\title{
Birds from northeastern Bogotá Savannah, Cundinamarca, Colombia
}

\author{
Fernando Castro-Vargas ${ }^{1}$, Yerson Cruz-Mendivelso ${ }^{1}$, Darwin Ortega-Chamorro ${ }^{1}$, Fredy Palacino- \\ Rodríguez ${ }^{2,3}$
}

1 Reserva Natural Ecoparque Sabana, Parque Jaime Duque, km 34 Autopista Norte Tocancipá, 251010, Cundinamarca department, Colombia. 2 Grupo de Investigación en Biología (GRIB), Departamento de Biología, Universidad El Bosque, Av. Cra. 9 No. 131A-02, 110121, Bogotá, Colombia. 3 Grupo de Investigación en Odonatos y otros artrópodos de Colombia (GINOCO), Centro de Investigación en Acarología, Calle 152B \# 55-45, 111321, Bogotá, Colombia.

Corresponding author: Fredy Palacino-Rodríguez, odonata17@hotmail.com

\begin{abstract}
The northeast area of the Bogotá Savannah (Cundinamarca, Colombia) is a region where biodiversity requires research because of few records in the current literature. We present a list of birds from the northeast area of the Bogotá Savannah. A systematic sample was conducted in the Ecoparque Sabana Nature Reserve (Tocancipá) to record the frequency of occurrence of bird species, while opportunistic observations were made at Parque Jaime Duque Nature Reserve (Tocancipá) and Aguablanca wetland (Sesquilé). A total of $820 \mathrm{~h}$ of sampling was done between February 2016 and June 2019. We provide a list with 128 species belonging to 37 families. Of these species, eight are newly recorded from the Bogotá Savannah; one species is considered Endangered and three are Near Threatened according to the Red List of the International Union for Conservation of Nature. The species richness found in our study supports the importance of protected areas in the northeast area of the Bogotá Savannah. Protected areas here help conserve the biodiversity of birds of the Andean region.
\end{abstract}

\section{Keywords}

Andean forest, Neotropical region, ornithological inventory, restored habitat, South America.

Academic editor: Flor Maria Las-Casas | Received 29 April 2020 | Accepted 17 September 2020 | Published 19 October 2020

Citation: Castro-Vargas F, Cruz-Mendivelso Y, Ortega-Chamorro D, Palacino-Rodríguez F (2020) Birds from northeastern Bogotá Savannah, Cundinamarca, Colombia. Check List 16 (5): 1375-1391. https://doi.org/10.15560/16.5.1375

\section{Introduction}

Colombia is one of the countries with the highest diversity of plants, mammals, amphibians, and birds in the world (Rangel 2006). The Colombian avifauna is composed of 1,941 species that make up $20 \%$ of all bird species in the world (Ayerbe-Quiñones 2018). Of these species, 974 have been reported in the Andean region (Rangel 2015). The Colombian Andean region is divided into 19 subregions, one of which is the Altiplano Cundiboyacense
(IGAC 1996). The Bogotá Savannah comprises the southern portion of this plateau and includes the city of Bogotá and nearby towns. The Bogotá Savannah is characterized by wetlands ecosystems $(2,550-2,600 \mathrm{~m}$ a.s.l.), xerophytic scrublands $(2,600-2,800 \mathrm{~m}$ a.s.1.), and para$\operatorname{mos}(3,300-4,200 \mathrm{~m}$ a.s.1.) (Calvachi 2012).

A total of 235 species of birds have been reported from the Bogotá Savannah, including 46 boreal migra- 
tory species, three endemic species (Rallus semiplumbeus P.L. Scatler, 1856, Synallaxis subpudica P.L. Sclater, 1874, and Cistothorus apolinari Chapman, 1914), four endemic subspecies (Ixobrychus exilis bogotensis (Chapman, 1914), Porphyriops melanops bogotensis (Vieillot, 1819), Eremophila alpestris peregrina (P.L. Sclater, 1855), and Chrysomus icterocephalus bogotensis Linnaeus, 1766), two species under global threat ( $R$. semiplumbeus and C. apolinari), and four species under local threat (Oxyura jamaicensis (Gmelin, 1789), Porphyriops melanops (Chapman, 1914), Pseudocolopteryx acutipennis (Sclater \& Salvin, 1873), and Eremophila alpestris (Linnaeus, 1758) (Stiles et al. 2017). Likewise, 188 species have been reported in the Bogotá wetlands (Echeverry-Galvis 2015), and 95 species have been recorded at the eastern forests from the Bogotá Protective Forest Reserve (BOBPFR) at the northeastern limit of the city (Peraza 2011). In addition, 400 species have been recorded in the Chingaza National Natural Park (PNN) (Linares-Romero et al. 2017).

Habitats in the Bogotá Savannah have been exposed to agriculture since 1500 (Etter and Van Wyngaarden 2000; Etter et al. 2008). According to Stiles et al. (2017), about $50 \%$ of the bird species in Bogotá and its surroundings have changed in their abundances over time in response to a combination of factors such as deforestation, exotic plantations, crops, climate change, and secondary succession in forests that have positively or negatively affected species. The habitats at the localities of our study are negatively affected by non-organic fertilizers, pesticides, cattle raising, deforestation, exotic plantations, crops such as corn and potatoes, and climate change (Rodríguez et al. 2006; Stiles et al. 2017). However, since 2016, the localities in our study have been positively influenced by the creation of artificial bodies of water, the planting of gardens and other afforestation patches with native vegetation, secondary succession in forests, continuous monitoring of bird populations (Stiles et al. 2017), and a reduction in hunting of some bird species, which was common in the past (Olivares 1969).

In this study, we present the results of four years (2016-2019) of systematic surveys in the Ecoparque Sabana Nature Reserve (ESNR) and opportunistic observations in the Parque Jaime Duque Nature Reserve (PJDNR), and in the Aguablanca wetland. We also present for the first time an updated list with important new records of birds in northeastern Bogotá Savannah. Besides presenting this list of the bird species, we discuss the relationship between the frequency of occurrence of species in the ESNR and the restoration process that is underway in this locality.

\section{Methods}

Study area. Ecoparque Sabana Nature Reserve (ESNR) and Parque Jaime Duque Nature Reserve (PJDNR) are two civil society nature reserves located at Parque Jaime
Duque, in Tocancipá municipality $\left(04^{\circ} 56^{\prime} 52^{\prime \prime} \mathrm{N}, 073^{\circ}\right.$ 57'47"W; Fig. 1) $33 \mathrm{~km}$ from Bogotá in Cundinamarca department. Aguablanca is a wetland in Sesquilé municipality $\left(05^{\circ} 02^{\prime} 43^{\prime \prime} \mathrm{N}, 073^{\circ} 47^{\prime} 50^{\prime \prime} \mathrm{W}\right.$; Fig. 1) $50 \mathrm{~km}$ from Bogotá, in Cundinamarca department. The average altitude in these localities is $2,600 \mathrm{~m}$ a.s.l., with wetlands, dry mountainous forests (bs-MB; Holdridge 1967), and lower Andean forests (Cortés et al. 1999). The annual median precipitation is $857 \mathrm{~mm}$, with a bimodal rain regime, and there is a wet season from April to June and from September to November. The mean annual temperature is $14.1{ }^{\circ} \mathrm{C}$, with an average of $80 \%$ relative humidity (IDEAM 2017; Climate-data.org 2018).

Parque Jaime Duque Nature Reserve (PJDNR, Fig. 2A) is part of the Tibito hill, and just like the Aguablanca wetland (Fig. 2B), shows mostly Andean forest with species such as Xylosma spiculifera G. Forst, 1786 (Corono), Duranta mutisii Linnaeus, 1753 (Espino Garbancillo), Myrcianthes leucoxyla (Ortega) McVaugh, 1800 (Arrayán), Myrsine guianensis (Aubl.) Kuntze, 1891 (Cucharo), bromelias, moss, hepatics, and lichens (Cortés et al. 1999; Pérez 2000).

Ecoparque Sabana Nature Reserve (ESNR; Fig. 2C, D) has two wetlands, which in 2016 were polluted and eutrophicated due to industrial and domestic pollution and intensive farming (Palacios and Safford 2002; Mora 2015). The main plant species that make up the vegetation in this reserve are Cenchrus cladestinus Hochst. ex Chiov. (Kikuyo), Eucalyptus spp., Acacia spp., Syzygium spp. (Eugenia), Genista monspessulana (L.) L.A.S. Johnson (French Broom), Ulex europaeus Linnaeus (Gorse), Eichornia crassipes (Martius) Solms (Water Hyacinth), Hydrocotyle ranunculoides L.F. (Floating Pennywort), and Typha latifolia (L.) Dulac (Broadleaf Cattail).

Data collection. We collected systematic and opportunistic observation data. In the PJDNR and Aguablanca, we carried out opportunistic observations due to the topography and dense vegetation in those areas. In the ESNR, we performed the inventory along a $4 \mathrm{~km}$ long transect. Along this transect, we made ten $200 \mathrm{~m}$ long by $50 \mathrm{~m}$ wide transects, which were selected because of the diversity of their vegetation structure and presence of bodies of water. Between these transects, we left 100 $\mathrm{m}$ long spaces where no sampling was conducted (Fig. 3). We estimated the frequency of occurrence as the number of samples where a given species was recorded divided by the total number of samplings (D'Angelo Neto et al. 1998). Two observers collected data during 41 months between February 2016 and June 2019 and did one-day expeditions each month (6 hours of sampling effort per day). Thus, we sampled for a total of 820 hours (410 hours per observer). Observations were made from 5:00 to 11:00 $\mathrm{h}$ (492 hours) and from 17:00 to 21:00 (328 hours).

The birds were visually identified using $10 \times 42$ binoculars and, when possible, photographic records were 


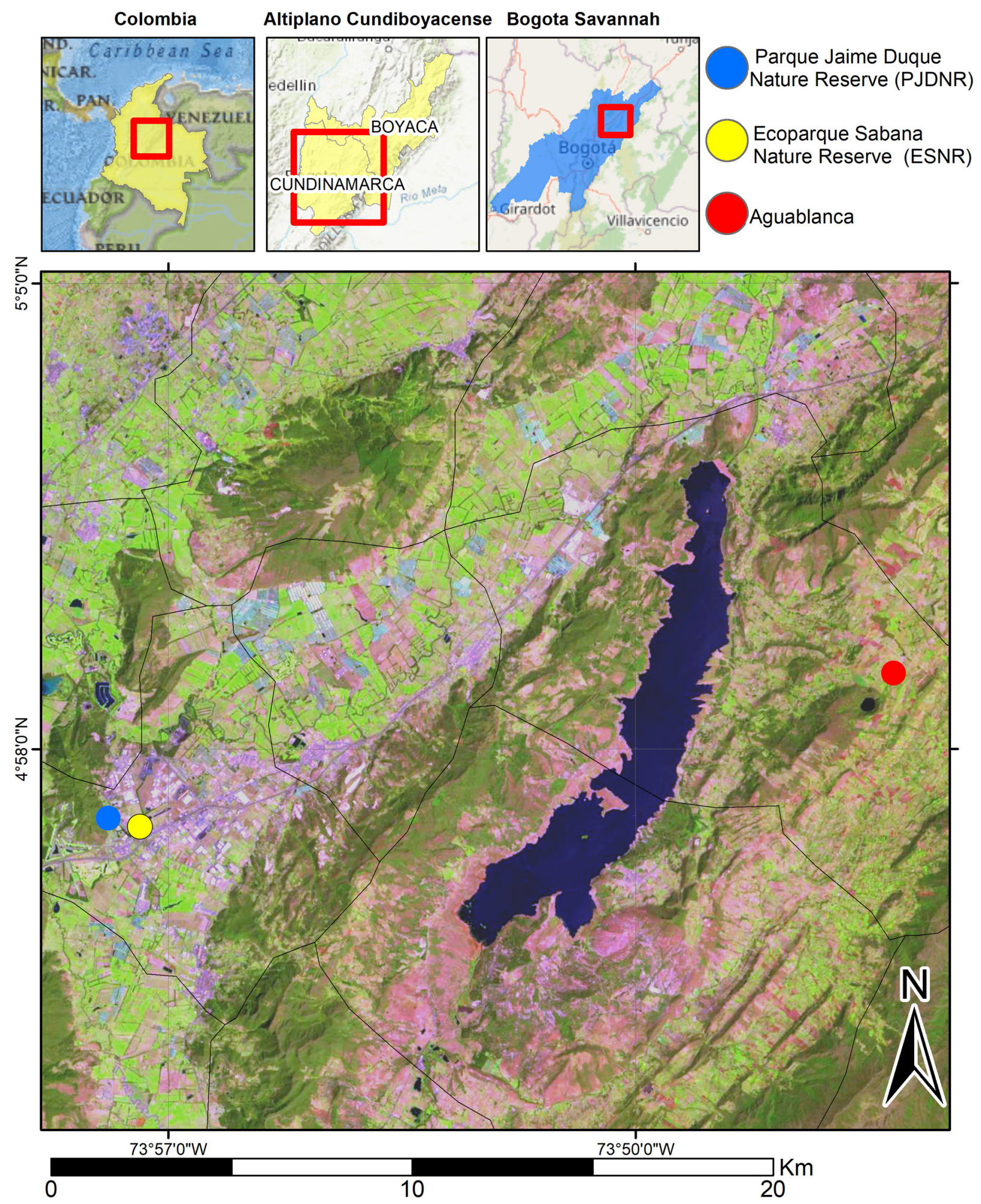

Figure 1. Map showing the locations in Tocancipá and Sesquilé municipalities, Cundinamarca department, Colombia.

taken using an $82 \times$ camera. Vocalizations were recorded with a Zoom H6 and SSH-6 Stereo Shotgun microphone recorder, and audio tracks are stored at XenoCanto (https://www.xeno-canto.org). Threatened species were determined according to the Red List of the International Union for Conservation of Nature's Red List (IUCN; https://www.iucnredlist.org) or BirdLife International $(2018,2019)$. Location of perch, reproductive events, and behavioral events such as flight, foraging, and intra- and interspecific interactions were also recorded. No specimens were collected during this study.

Taxonomy and nomenclature follow the American Classification Committee (https://www.museum.lsu.edu/ $\sim$ Remsen/SACCBaseline.htm). To verify the identity of the species, we used the taxonomic keys by ABO (2000), Hilty and Brown (2001), and Ayerbe-Quiñones (2018). Geographic distribution data follows the Neotropical Birds Online (2019). In order to compare our records 

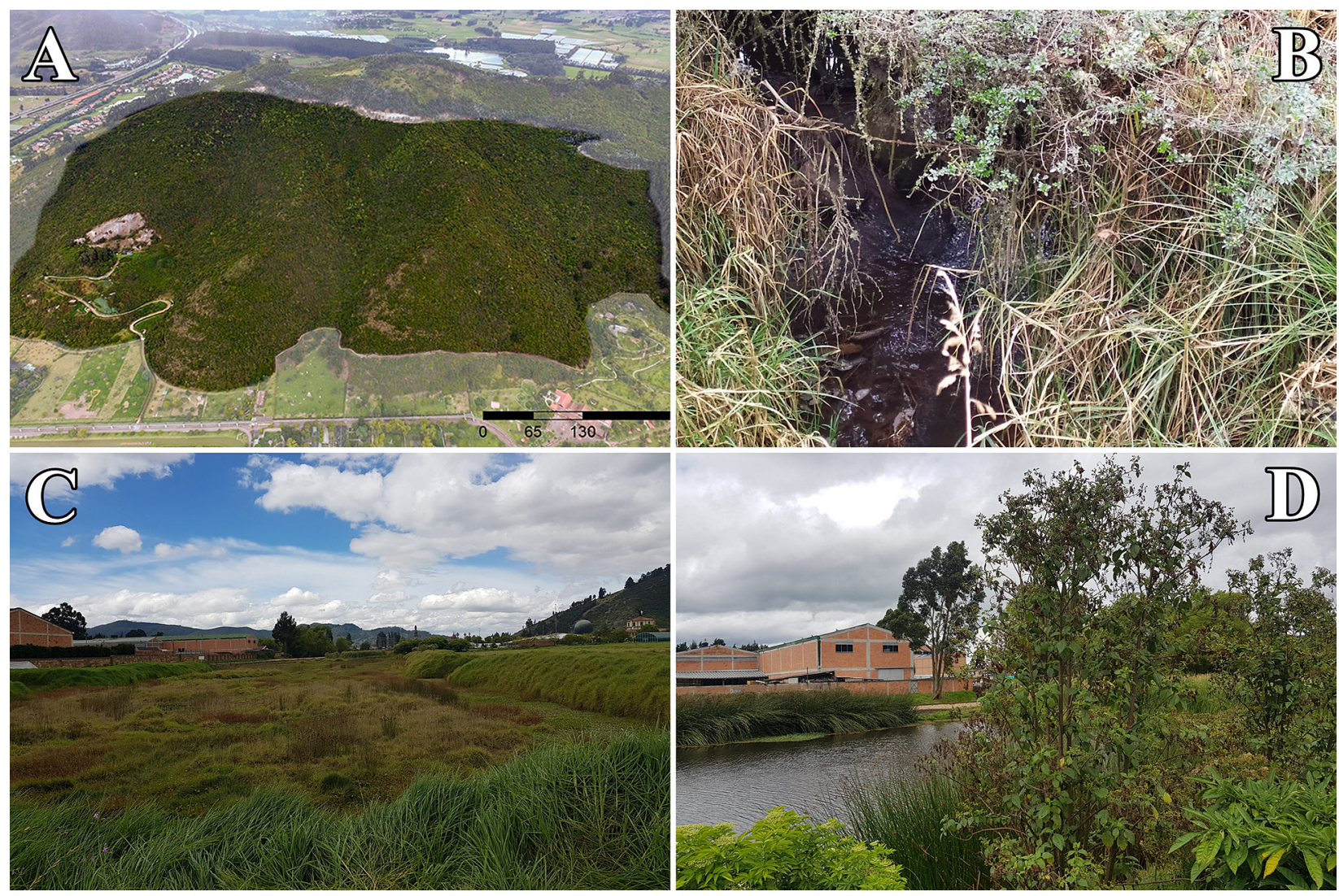

Figure 2. Localities sampled in the northeastern area of Bogotá Savannah. A. Parque Jaime Duque Nature Reserve (PJDNR; source: Orthophoto ESNR). B. Aguablanca Wetlands. C-D. Ecoparque Sabana Nature Reserve (ESNR; source: Orthophoto ESNR).

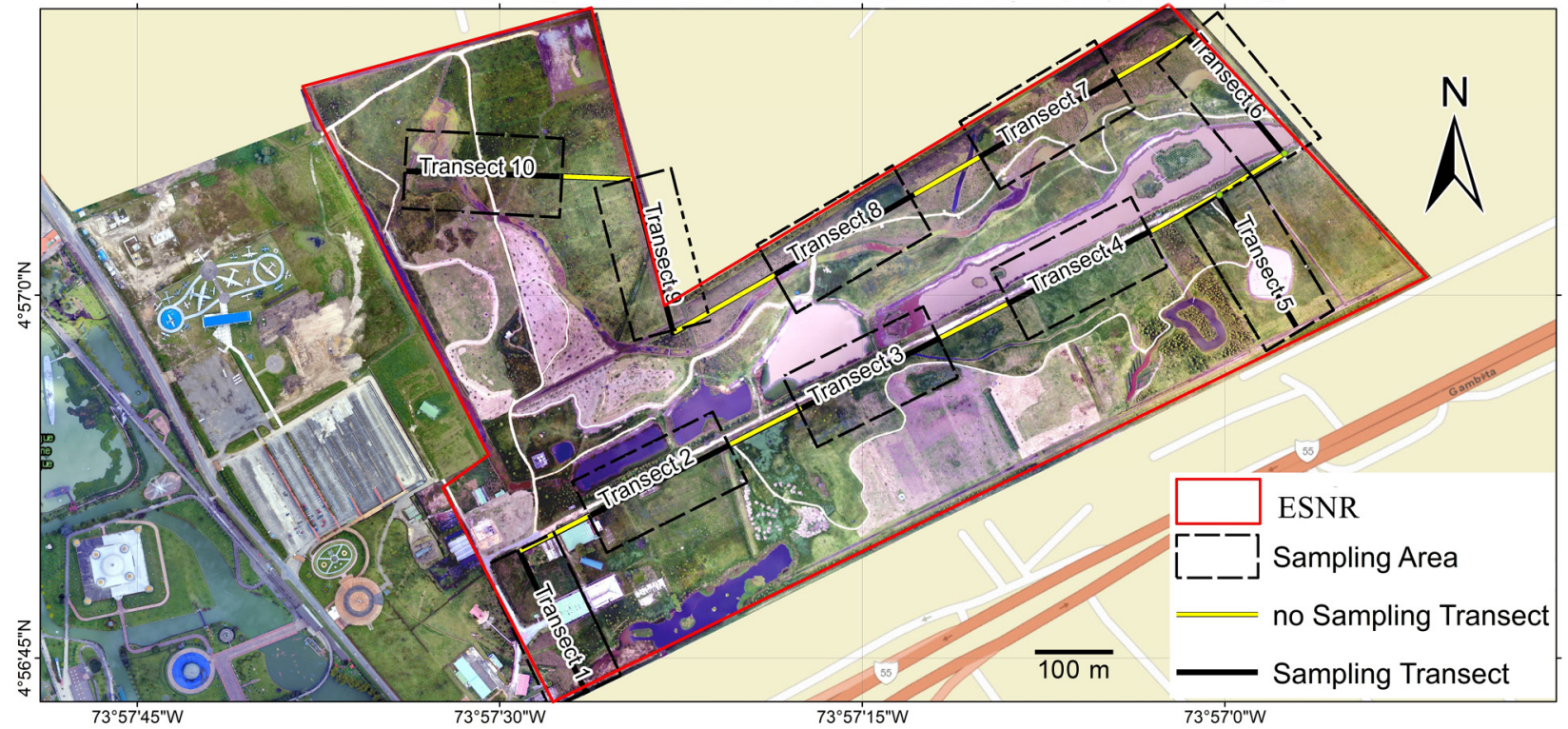

Figure 3. Sampling methods in Ecoparque Sabana Nature Reserve (ESNR), Tocancipá municipality.

with list of species from similar localities representative of the regional conditions in mountain areas of eastern Colombian habitats, we analyzed the reports of Bogotá wetlands (Echeverry-Galvis 2015), the Bogotá Protective Forest Reserve (BOBPFR; Peraza 2011), and the Chingaza National Natural Park (Linares-Romero et al. 2017). Our study is the first inventory of birds for the ESNR, PJDNR, and Aguablanca wetland.

\section{Results}

We observed 128 species of birds belonging to 37 families. Through systematic sampling, we recorded 102 species, of which 89 ( $69 \%$ of the total) were exclusively from ESNR. The families with highest richness were Tyrannidae (15 spp.), Parulidae (10 spp.), and Thraupidae (10 spp.). Our study reports eight species that were not previously reported from northeastern Bogotá Savannah: 
Table 1. Checklist of bird species of northeast of Bogotá Savannah. *Species only in Parque Jaime Duque Nature Reserve; ** Species not recorded in PNN Chingaza; 'Species not recorded in Bosque Oriental from Bogotá Protective Forest Reserve; 'Species not recorded in Bogotá wetlands. S: Sesquilé, T: Tocancipá, \%O: percentage of occurrences.

\begin{tabular}{|c|c|c|c|c|c|c|c|c|c|}
\hline \multirow{2}{*}{ Taxon name } & \multirow{2}{*}{ English name } & \multirow{2}{*}{ UICN } & \multirow{2}{*}{ Xeno-canto records } & \multicolumn{2}{|c|}{ Locality } & \multicolumn{4}{|c|}{ Percentage occurrence } \\
\hline & & & & $S$ & $\mathbf{T}$ & 2016 & 2017 & 2018 & 2019 \\
\hline \multicolumn{10}{|l|}{ Anseriformes } \\
\hline \multicolumn{10}{|l|}{ Anatidae } \\
\hline Spatula discors (Linnaeus, 1766) ${ }^{* * 0}$ & Blue-winged Teal & LC & XC501464 & $x$ & $x$ & 60 & 100 & 100 & 100 \\
\hline Anas andium (Conover, 1941) ${ }^{\circ}$ & Andean Teal & LC & & & $x$ & - & - & 8.3 & - \\
\hline Oxyura jamaicensis andina $(\text { Gmelin } 1789)^{\circ}$ & Ruddy Duck & LC & & & $x$ & - & 60 & 100 & 100 \\
\hline \multicolumn{10}{|l|}{ Galliformes } \\
\hline \multicolumn{10}{|l|}{ Cracidae } \\
\hline Penelope montagnii (Bonaparte, 1756) • & Adean Guan & $\mathrm{LC}$ & & & $X^{*}$ & - & - & - & - \\
\hline \multicolumn{10}{|l|}{ Odontophoridae } \\
\hline Colinus cristatus (Linnaeus, 1758)** & Crested Bobwhite & LC & & $x$ & $x$ & - & 8.3 & - & - \\
\hline \multicolumn{10}{|l|}{ Podicipediformes } \\
\hline \multicolumn{10}{|l|}{ Podicipedidae } \\
\hline Podilymbus podiceps (Linnaeus, 1758)*** & Pied-billed Grebe & LC & XC575479 & & $x$ & - & 66.6 & 100 & 100 \\
\hline Columbiformes & & & & & & & & & \\
\hline Columbidae & & & & & & & & & \\
\hline Columba livia Linnaeus, $1758^{* * 0}$ & Rock dove & LC & & & $x$ & - & - & 8.3 & - \\
\hline Patagioenas fasciata (Say, 1822) • & Northern Band-tailed Pigeon & LC & & $x$ & $X^{*}$ & - & - & - & - \\
\hline Zenaida auriculata (Des Murs, 1847)• & Eared Dove & LC & & $x$ & $x$ & 100 & 100 & 100 & 100 \\
\hline Cuculiformes & & & & & & & & & \\
\hline Cuculidae & & & & & & & & & \\
\hline Crotophaga major Gmelin, $1788^{* * *}$ & Greater Ani & LC & & & $x$ & - & - & 8.3 & - \\
\hline Coccyzus americanus (Linnaeus, 1758) & Yellow-billed Cuckoo & LC & & $x$ & $x$ & - & 8.3 & 8.3 & - \\
\hline Caprimulgiformes & & & & & & & & & \\
\hline Caprimulgidae & & & & & & & & & \\
\hline Chordeiles minor (Forster, 1771) ${ }^{\circ}$ & Common Nighthawk & $\mathrm{LC}$ & & & $x$ & - & 8.3 & 8.3 & 8.3 \\
\hline Systellura longirostris (Bonaparte, 1825) & Band-winged Nightjar & LC & & & $x$ & - & - & 8.3 & 8.3 \\
\hline Apodiformes & & & & & & & & & \\
\hline Trochilidae & & & & & & & & & \\
\hline Colibri coruscans (Gould, 1846) • & Sparkling Violetear & LC & XC446943 & $x$ & $x$ & 75 & 91.6 & 91.6 & 91.6 \\
\hline Lesbia nuna (Lesson, 1832)• & Green-tailed Trainbearer & LC & XC582123 & $x$ & $x$ & - & - & 8.3 & 8.3 \\
\hline Eriocnemis vestita (Lesson, 1839)• & Glowing Puffleg & LC & & $x$ & $x$ & - & - & - & - \\
\hline Aglaeactis cupripennis (Bourcier, 1843) & Shining Sunbeam & LC & & $x$ & & - & - & - & - \\
\hline Chaetocercus mulsant (Bourcier, 1843) & White-bellied Woodstar & LC & & & $x$ & - & - & 8.3 & - \\
\hline Gruiformes & & & & & & & & & \\
\hline Rallidae & & & & & & & & & \\
\hline Rallus semiplumbeus P.L. Scatler, 1856 & Bogotá Rail & EN & XC506788 & & $x$ & - & - & - & 8.3 \\
\hline Porphyrio martinica (Linnaeus, 1766)**० & Purple Gallinule & LC & & & $x$ & - & 8.3 & 8.3 & 8.3 \\
\hline Mustelirallus erythrops (P.L. Sclater, 1867)** & Paint-billed Crake & LC & & & $x$ & - & 8.3 & - & - \\
\hline Porphyriops melanops bogotensis (Vieillot, 1819) ${ }^{* * *}$ & Spot-flanked Gallinule & LC & XC470011 & & $x$ & 8.3 & 100 & 100 & 100 \\
\hline Porzana carolina (Linnaeus, 1758$)^{\circ}$ & Sora & LC & & & $x$ & 8.3 & 16.6 & 16.6 & 16.6 \\
\hline Gallinula galeata (Lichtenstein, 1818) ${ }^{\circ}$ & Common Gallinule & LC & & & $x$ & 63.6 & 100 & 100 & 100 \\
\hline Fulica americana Gmelin, $1789^{\circ}$ & American Coot & $\mathrm{LC}$ & XC575486 & & $x$ & 63.6 & 100 & 100 & 100 \\
\hline Charadriiformes & & & & & & & & & \\
\hline Charadriidae & & & & & & & & & \\
\hline Vanellus chilensis (Molina, 1782) ${ }^{\circ}$ & Southern Lapwing & LC & XC460550 & & $x$ & 63.6 & 100 & 100 & 100 \\
\hline Charadrius vociferus Linnaeus, $1758^{* * 0}$ & Killdeer & LC & XC460580 & & $x$ & 63.6 & 100 & 100 & 100 \\
\hline Scolopacidae & & & & & & & & & \\
\hline Bartramia longicauda (Bechstein, 1812)**o & Upland Sandpiper & LC & & & $x$ & - & - & - & 8.3 \\
\hline Calidris melanotos (Vieillot, 1819) & Pectoral Sandpiper & $\mathrm{LC}$ & & & $x$ & - & - & - & 8.3 \\
\hline Gallinago nobilis P.L. Scater, $1856^{\circ}$ & Noble Snipe & NT & XC558706 & & $x$ & 63.6 & 100 & 100 & 100 \\
\hline Actitis macularius (Linnaeus, 1766$)^{\circ}$ & Spotted Sandpiper & $\mathrm{LC}$ & & & $x$ & 18.2 & 50 & 50 & 50 \\
\hline Tringa solitaria Wilson, $1813^{\circ}$ & Solitary Sandpiper & LC & XC460565 & & $x$ & 36.6 & 41.6 & 41.6 & 41.6 \\
\hline Tringa melanoleuca (Gmelin, 1789) ${ }^{\circ}$ & Greater Yellowlegs & LC & & & $x$ & 8.3 & 8.3 & 8.3 & 8.3 \\
\hline Tringa flavipes (Gmelin, 1789) ${ }^{\circ}$ & Lesser Yellowlegs & LC & & & $x$ & 25 & 25 & 25 & 25 \\
\hline Jacanidae & & & & & & & & & \\
\hline Jacana jacana (Linnaeus, 1766)*** & Wattled Janaca & LC & & & $x$ & - & 8.3 & - & - \\
\hline Laridae & & & & & & & & & \\
\hline Phaetusa simplex (Gmelin, 1789)*** & Large-billed Tern & $\mathrm{LC}$ & & & $x$ & - & 8.3 & 8.3 & - \\
\hline Suliformes & & & & & & & & & \\
\hline Phalacrocoracidae & & & & & & & & & \\
\hline Phalacrocorax brasilianus (Gmelin, 1789)**० & Neotropic Cormorant & LC & & & $x$ & - & 91.6 & 91.6 & 50 \\
\hline
\end{tabular}




\begin{tabular}{|c|c|c|c|c|c|c|c|c|c|}
\hline \multirow{2}{*}{ Taxon name } & \multirow{2}{*}{ English name } & \multirow{2}{*}{ UICN } & \multirow{2}{*}{ Xeno-canto records } & \multicolumn{2}{|c|}{ Locality } & \multicolumn{4}{|c|}{ Percentage occurrence } \\
\hline & & & & $s$ & $\mathbf{T}$ & 2016 & 2017 & 2018 & 2019 \\
\hline \multicolumn{10}{|l|}{ Pelecaniformes } \\
\hline \multicolumn{10}{|l|}{ Ardeidae } \\
\hline Nycticorax nycticorax (Linnaeus, 1758) & Black-crowned Night-heron & $\mathrm{LC}$ & & & $x$ & 8.3 & 8.3 & 8.3 & 8.3 \\
\hline Butorides striata (Linnaeus, 1758)**•• & Striated Heron & $\mathrm{LC}$ & & & $x$ & 45.5 & 66.6 & 66.6 & 91.7 \\
\hline Bubulcus ibis (Linnaeus, 1758) ${ }^{\bullet \bullet}$ & Cattle Egret & $\mathrm{LC}$ & & $x$ & $x$ & 100 & 100 & 100 & 100 \\
\hline Ardea alba Linnaeus, $1758^{\circ}$ & Great Egret & $\mathrm{LC}$ & & & $x$ & 54.5 & 83.3 & 83.3 & 100 \\
\hline Syrigma sibilatrix (Temminck, 1824) ${ }^{\circ}$ & & $\mathrm{LC}$ & & & $x$ & - & - & 8.3 & 8.3 \\
\hline Egretta caerulea (Linnaeus, 1758) ${ }^{\circ}$ & Little Blue Heron & $\mathrm{LC}$ & & & $x$ & 8.3 & 8.3 & 8.3 & 8.3 \\
\hline \multicolumn{10}{|l|}{ Threskiornithidae } \\
\hline Phimosus infuscatus (Lichtenstein, 1823) & Bare-faced lbis & LC & & & $x$ & - & - & 16.6 & 41.7 \\
\hline \multicolumn{10}{|l|}{$\begin{array}{l}\text { Cathartiformes } \\
\text { Cathartidae }\end{array}$} \\
\hline Coragyps atratus (Bechstein, 1793) • & Black Vultutre & $\mathrm{LC}$ & & $x$ & $x$ & 75 & 75 & 75 & 41.7 \\
\hline \multicolumn{10}{|l|}{ Pandionidae } \\
\hline Pandion haliaetus (Linnaeus, 1758) ${ }^{\circ}$ & Osprey & $\mathrm{LC}$ & & & $x$ & 8.3 & 8.3 & 8.3 & - \\
\hline Accipitriformes & & & & & & & & & \\
\hline Accipitridae & & & & & & & & & \\
\hline Elanus leucurus (Vieillot, 1818) • & White-tailed Kite & $\mathrm{LC}$ & & $x$ & $x$ & 91.6 & 91.6 & 91.6 & 58.3 \\
\hline Elanoides forficatus (Linnaeus, 1758) ${ }^{\circ}$ • & Swallow-tailed Kite & $\mathrm{LC}$ & & & $x^{*}$ & - & - & - & - \\
\hline Accipiter striatus Vieillot, $1808^{* * \bullet \bullet}$ & Sharp-shinned Hawk & $\mathrm{LC}$ & & $x$ & & - & - & - & - \\
\hline Rupornis magnirostris (J.F. Gmelin, 1788) • & Roadside Hawk & $\mathrm{LC}$ & XC461499 & $x$ & $x$ & 75 & 75 & 75 & 83.3 \\
\hline Buteo platypterus (Vieillot, 1823) & Broad-winged-Hawk & $\mathrm{LC}$ & & & $x$ & 8.3 & 8.3 & 8.3 & 8.3 \\
\hline Buteo swainsoni Bonaparte, $1838^{\circ}$ & Swaison's Hawk & $\mathrm{LC}$ & & & $x$ & 8.3 & 8.3 & 8.3 & 8.3 \\
\hline Strigiformes & & & & & & & & & \\
\hline Tytonidae & & & & & & & & & \\
\hline Tyto alba (Scopoli, 1769) • & Barn 0wl & $\mathrm{LC}$ & & $x$ & $x$ & - & - & 100 & 100 \\
\hline Strigidae & & & & & & & & & \\
\hline Megascops choliba (Vieillot, 1817) ${ }^{\circ}$ & Tropical Screech-Owl & $\mathrm{LC}$ & & & $x$ & - & - & 8.3 & 8.3 \\
\hline Asio clamator (Vieillot, 1808)**०• & Striped Owl & $\mathrm{LC}$ & & & $x$ & - & 8.3 & 8.3 & 8.3 \\
\hline Asio flammeus bogotensis Chapman, 1915***• & Short-eared 0wl & $\mathrm{LC}$ & & & $x$ & - & 16.6 & 16.6 & 16.6 \\
\hline Piciformes & & & & & & & & & \\
\hline Picidae & & & & & & & & & \\
\hline Dryobates fumigatus (d'Orbigny, 1840) • & Smoky-brown Woodpecker & $\mathrm{LC}$ & & & $x^{*}$ & - & - & - & - \\
\hline Colaptes rivolii (Boissonneau, 1840$)^{\circ}$ & Crimson-mantled Woodpecker & LC & & $x$ & & - & - & - & - \\
\hline Falconiformes & & & & & & & & & \\
\hline Falconidae & & & & & & & & & \\
\hline Caracara cheriway (Jacquin, 1784)**०• & Crested caracara & $\mathrm{LC}$ & & & $x$ & - & - & - & 16.6 \\
\hline Falco sparverius Linnaeus, $1758^{\circ}$ & American Kestrel & $\mathrm{LC}$ & & $x$ & $x$ & - & 25 & 41.6 & 25 \\
\hline Falco columbarius Linnaeus, $1758^{\circ}$ & Merlin & $\mathrm{LC}$ & & & $x$ & - & - & 8.3 & 16.7 \\
\hline Falco peregrinus Tunstall, $1771^{* * \circ}$ & Peregrine Falcon & $\mathrm{LC}$ & & & $x$ & - & - & 33.3 & 66.6 \\
\hline Psittaciformes & & & & & & & & & \\
\hline Psittacidae & & & & & & & & & \\
\hline Forpus conspicillatus (Lafresnaye, 1848) ${ }^{\circ}$ & Spectacled Parrotlet & $\mathrm{LC}$ & & & $x$ & - & - & 8.3 & - \\
\hline Grallariidae & & & & & & & & & \\
\hline Grallaria squamigera Lafresnaye, $1842^{\circ}{ }^{\circ}$ & Undulated Antpitta & $\mathrm{LC}$ & & $x$ & & - & - & - & - \\
\hline Grallaria ruficapilla Lafresnaye, $1842^{\circ}$ & Chestnut-crowned Antpitta & $\mathrm{LC}$ & & $x$ & & - & - & - & - \\
\hline Grallaria rufula Lafresnaye, $1843^{\circ} \bullet$ & Rufous Antpitta & LC & & $x$ & & - & - & - & - \\
\hline Rhinocryptidae & & & & & & & & & \\
\hline Scytalopus griseicollis (Lafresnaye,1840)• & Pale-bellied Tapaculo & $\mathrm{LC}$ & & & $x^{*}$ & - & - & - & - \\
\hline Furnariidae & & & & & & & & & \\
\hline Synallaxis subpudica P.L. Sclater, $1874^{\bullet}$ & Silvery-throated Spinetail & $\mathrm{LC}$ & XC462131 & & $x$ & - & - & 8.3 & - \\
\hline Tyrannidae & & & & & & & & & \\
\hline Pyrrhomyias cinnamomeus (d'Orbigny \& Lafresnaye, 1837)" & Cynnamon Flycatcher & $\mathrm{LC}$ & & $x$ & & - & - & - & - \\
\hline Elaenia frantzii Lawrence, $1865^{\circ}{ }^{\circ}$ & Mountain Elaenia & $\mathrm{LC}$ & & $x$ & $x^{*}$ & - & - & - & - \\
\hline Mecocerculus leucophrys (d'Orbigny \& Lafresnaye, 1837) • & White-throated Tyrannulet & $\mathrm{LC}$ & XC465233 & $x$ & $x$ & - & 8.3 & 8.3 & 8.3 \\
\hline Serpophaga cinerea (Tschudi, 1844)***• & Torrent Tyrannulet & $\mathrm{LC}$ & & & $x$ & - & - & - & 8.3 \\
\hline Machetornis rixosa (Vieillot, 1819)*** & Cattle Tyrant & $\mathrm{LC}$ & & & $x$ & - & - & 8.3 & 8.3 \\
\hline Myiodynastes luteiventris (Sclater, 1859)**o & Sulphur-belled Flycatcher & $\mathrm{LC}$ & & & $x$ & - & - & 8.3 & 8.3 \\
\hline Tyrannus melancholicus Vieillot, $1819^{\circ} \bullet$ & Tropical Kingbird & $\mathrm{LC}$ & & $x$ & $x$ & 83.3 & 83.3 & 83.3 & 83.3 \\
\hline Tyrannus savana Daudin, $1802^{\circ}$ & Fork-tailed Flycatcher & $\mathrm{LC}$ & & $x$ & $x$ & - & 25 & 25 & 25 \\
\hline Tyrannus tyrannus (Linnaeus, 1758) & Eastern Kingbird & $\mathrm{LC}$ & & & $x$ & - & 16.6 & 16.6 & 16.6 \\
\hline Myiarchus crinitus (Linnaeus, 1758)**o & Great Crested Flycatcher & LC & & & $x$ & - & 8.3 & 8.3 & - \\
\hline Pyrocephalus rubinus (Baddaert, 1783)**• & Vermillion Flycatcher & LC & & & $x$ & - & - & - & - \\
\hline Sayornis nigricans (Swainson, 1827) ${ }^{\circ}$ & Black Phoebe & $\mathrm{LC}$ & & & $x$ & 8.3 & 8.3 & 8.3 & 16.7 \\
\hline Contopus cooperi (Nuttall, 1831) & Olive-sided Flycatcher & NT & & & $x$ & - & - & 8.3 & - \\
\hline Contopus virens (Linnaeus, 1766)**o & Eastern Wood-Pewee & $\mathrm{LC}$ & & & $x$ & - & 25 & 25 & 25 \\
\hline
\end{tabular}




\begin{tabular}{|c|c|c|c|c|c|c|c|c|c|}
\hline \multirow{2}{*}{ Taxon name } & \multirow{2}{*}{ English name } & \multirow{2}{*}{ UICN } & \multirow{2}{*}{ Xeno-canto records } & \multicolumn{2}{|c|}{ Locality } & \multicolumn{4}{|c|}{ Percentage occurrence } \\
\hline & & & & $S$ & $T$ & 2016 & 2017 & 2018 & 2019 \\
\hline \multicolumn{10}{|l|}{ Vireonidae } \\
\hline Vireo olivaceus (Linnaeus, 1766)**o & Red-Eyed Vireo & LC & & & $x$ & - & 8.3 & 16.6 & 33.3 \\
\hline \multicolumn{10}{|l|}{ Hirundinidae } \\
\hline Orochelidon murina (Cassin, 1853) & Brown-bellied Swallow & LC & & $x$ & $x$ & 91.6 & 100 & 100 & 100 \\
\hline Progne tapera (Linnaeus, 1766$)^{\circ}$ & Brown-chested Martin & LC & & & $x$ & - & - & 25 & 8.3 \\
\hline Progne subis (Linnaeus, 1758)**॰ & Purple Martin & LC & & & $x$ & - & - & 8.3 & - \\
\hline Riparia riparia (Linnaeus, 1758) ${ }^{\circ}$ & Bank Swallow & LC & & & $x$ & 25 & 25 & 25 & 25 \\
\hline Hirundo rustica Linnaeus, 1758 & Barn Swallow & LC & & & $x$ & 25 & 25 & 25 & 25 \\
\hline Petrochelidon pyrrhonota (Vieillot, 1817)**o & Cliff Swallow & LC & & & $x$ & 16.6 & 16.6 & 16.6 & 16.6 \\
\hline \multicolumn{10}{|l|}{ Troglodytidae } \\
\hline Troglodytes aedon Vieillot, $1809^{\star}$ & House Wren & LC & & $x$ & $x$ & 81.8 & 100 & 100 & 100 \\
\hline \multicolumn{10}{|l|}{ Turdidae } \\
\hline Catharus ustulatus (Nuttall, 1840) & Russet-backed Thrush & LC & & & $x$ & - & - & 8.3 & 8.3 \\
\hline Turdus fuscater d'Orbigny \& Lafresnaye, $1837^{\star}$ & & LC & & $x$ & $x$ & 90.9 & 100 & 100 & 100 \\
\hline \multicolumn{10}{|l|}{ Mimidae } \\
\hline Mimus gilvus (Vieillot, 1808) & Tropical Mockingbird & LC & XC579873 & $x$ & $x$ & - & 66.6 & 66.6 & 83.3 \\
\hline \multicolumn{10}{|l|}{ Fringillidae } \\
\hline Spinus spinescens (Bonaparte, 1850$)^{\circ}$ & Andean Siskin & LC & & & $x$ & 100 & 100 & 100 & 100 \\
\hline Spinus psaltria $\left(\right.$ Say, 1822) ${ }^{* * \circ}$ & Lesser Goldfinch & LC & & $x$ & $x$ & 75 & 75 & 75 & 75 \\
\hline \multicolumn{10}{|l|}{ Passerellidae } \\
\hline Arremon assimilis (Boissonneau, 1840) ${ }^{\circ}$ & Grey-browed Brush Finch & NE & XC465062 & & $x^{*}$ & - & - & - & - \\
\hline Zonotrichia capensis (Statius Müller, 1776)` & Rufous-collared Sparrow & LC & XC446944 & $x$ & $x$ & 100 & 100 & 100 & 100 \\
\hline Atlapetes schistaceus (Boissonneau, 1840) & Slaty Brush-finch & LC & & $x$ & $x^{*}$ & - & - & - & - \\
\hline Atlapetes pallidinucha (Boissonneau, 1840$)^{\bullet}$ & Pale naped Brush-finch & LC & XC465239 & $x$ & $X^{*}$ & - & - & - & - \\
\hline \multicolumn{10}{|l|}{ Icteridae } \\
\hline Sturnella magna (Linnaeus, 1758) • & Eastern Meadowlark & NT & XC461497 & $x$ & $x$ & 100 & 100 & 100 & 100 \\
\hline Icterus chrysater (Lesson, 1844) ${ }^{\circ}$ & Yellow-backed oriole & LC & & $x$ & $x$ & 8.3 & 8.3 & 8.3 & 8.3 \\
\hline Molothrus bonariensis (Gmelin, 1789)**o & Shiny Cowbird & LC & & & $x$ & 91.6 & 91.6 & 91.6 & 75 \\
\hline Quiscalus lugubris Swainson, $1838^{* * 0}$ & Carib Grackle & LC & & & $x$ & - & 8.3 & 8.3 & 8.3 \\
\hline Gymnomystax mexicanus (Linnaeus, 1766$)^{\circ}$ & Oriole Blackbird & LC & XC572491 & & $x$ & - & - & 8.3 & - \\
\hline Chrysomus icterocephalus bogotensis (Linnaeus, 1766) ${ }^{* * 0}$ & Yellow-hooded Blackbird & LC & XC513503 & & $x$ & 63.6 & 91.6 & 91.6 & 91.6 \\
\hline \multicolumn{10}{|l|}{ Parulidae } \\
\hline Parkesia noveboracensis (Gmelin, 1789)*** & Northern waterthrush & LC & & & $x$ & - & - & - & 8.3 \\
\hline Protonotaria citrea (Boddaert, 1783)**0 & Prothonotary warbler & LC & & & $x$ & - & - & - & 8.3 \\
\hline Leiothlypis peregrina (Wilson, 1811)**o & Tennessee Warbler & LC & & & $x$ & - & - & 8.3 & 8.3 \\
\hline Geothlypis philadelphia (Wilson, 1810) & Mourning Warbler & LC & & & $x$ & - & - & 8.3 & 8.3 \\
\hline Setophaga ruticilla (Linnaeus, 1758) ${ }^{\circ}$ & American Redstart & LC & & & $x$ & - & 8.3 & 8.3 & 8.3 \\
\hline Setophaga cerulea (Wilson, 1810) ${ }^{\circ}$ & Cerulean Warbler & NT & & & $x$ & - & 8.3 & - & - \\
\hline Setophaga fusca (Müller, 1776) ${ }^{\circ}$ & Blackburnian Warbler & LC & & & $x$ & - & 8.3 & 8.3 & 8.3 \\
\hline Setophaga petechia (Linnaeus, 1766$)^{\circ}$ & Yellow Warbler & LC & & & $x$ & - & - & 8.3 & 8.3 \\
\hline Myiothlypis nigrocristata (Lafresnaye, 1840$)^{\circ} \bullet$ & Black-crested Warbler & LC & XC484799 & & $x^{*}$ & - & - & - & - \\
\hline 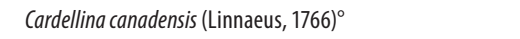 & Canada Warbler & LC & & & $x$ & - & 8.3 & 8.3 & 8.3 \\
\hline Cardinalidae & & & & & & & & & \\
\hline Piranga rubra (Linnaeus, 1758$)^{\circ}$ & Summer Tanager & LC & & & $x$ & - & 8.3 & 8.3 & 8.3 \\
\hline Piranga olivacea (Gmelin, 1789)**o & Scarlet Tanager & LC & & & $x$ & - & 8.3 & 8.3 & 8.3 \\
\hline Pheucticus aureoventris (d'Orbigny \& Lafresnaye, 1837) ${ }^{* * 0}$ & Black-backed Grosbeak & LC & & & $x^{*}$ & - & - & - & - \\
\hline Thraupidae & & & & & & & & & \\
\hline Conirostrum rufum Lafresnaye, $1843^{\circ}$ & Rufous-browed Conebill & LC & & & $X^{*}$ & - & - & - & - \\
\hline Sicalis flaveola (Linnaeus, 1766) ${ }^{* * 0}$ & Saffron Finch & LC & & & $x$ & - & 8.3 & 8.3 & 16.7 \\
\hline Sicalis luteola bogotensis Sparrman, $1789 * * \circ$ & Grassland Yellow-finch & LC & XC513507 & $x$ & $x$ & 100 & 100 & 100 & 91.7 \\
\hline Diglossa humeralis (Fraser, 1840) & Black Flowerpiercer & LC & XC465249 & $x$ & $x$ & - & - & 8.3 & 8.3 \\
\hline Diglossa sittoides (d'Orbigny \& Lafresnaye, 1837)*** & Rusty Flowerpiercer & LC & & & $x$ & 8.3 & 8.3 & 8.3 & 8.3 \\
\hline Sporophila luctuosa (Lafresnaye, 1843)**० & Black-and-white Seedeater & LC & & $x$ & & - & - & - & - \\
\hline Pipraeidea melanonota (Vieillot, 1819)• & Fawn-breasted Tanager & LC & & & $x^{*}$ & - & - & - & - \\
\hline Dubusia taeniata (Boissonneau, 1840)` & Buff-breasted Mountain-Tanager & LC & & & $X^{*}$ & - & - & - & - \\
\hline Anisognathus igniventris (d'Orbigny \& Lafresnaye, 1837) & Fire-bellied Mountain-tanager & LC & & $x$ & & - & - & - & - \\
\hline Thraupis episcopus (Linnaeus, 1766) ${ }^{\circ}$ & Blue-gray Tanager & $\mathrm{LC}$ & & & $x$ & - & 8.3 & 8.3 & 8.3 \\
\hline
\end{tabular}

Bartramia longicauda (Bechstein, 1812), Caracara cheriway Jacquin, 1784, Charadrius vociferus Linnaeus, 1758, Myiodynastes luteiventris Sclater, 1859, Progne subis Linnaeus, 1758, Progne tapera Linnaeus, 1766, Setophaga cerulea (Wilson, 1810), and Syrigma sibilatrix Temminck, 1824.
Zenaida auriculata (Des Murs, 1847), Bubulcus ibis (Linnaeus, 1758), Spinus spinescens (Bonaparte, 1850), Zonotrichia capensis (Müller, 1776), and Sturnella magna (Linnaeus, 1758) were recorded in all samples from 2016 to 2019. Spatula discors (Linnaeus, 1766), Porphyriops melanops bogotensis (Vieillot, 1819), 
Gallinula galeata (Lichtenstein, 1818), Fulica americana (Gmelin, 1789), Vanellus chilensis (Molina, 1782), Charadrius vociferus (Linnaeus, 1758), Gallinago nobilis P.L. Scater, 1856, Orochelidon murina (Cassin, 1853), Troglodytes aedon Vieillot, 1809, and Turdus fuscater d'Orbigny \& Lafresnaye, 1837 were recorded in all samples from 2017 to 2019.

We found that IUCN categorizes $122 \mathrm{spp}$. as Least Concern, and four species as Near Threatened. In the latter category are Gallinago nobilis, Sturnella magna, Contopus cooperi Nuttall, 1831, and Setophaga cerulea (Wilson, 1810). Rallus semiplumbeus is categorized as Endangered. Arremon assimilis (Boissonneau, 1840) has not been evaluated (Table 1). Information on these species and some natural history aspects are provided below. We also present some noteworthy records that include rarely seen species, migrants with few records, and important species that arrived after the restoration actions in the ESNR.

\section{New records from the northeastern Bogotá Savannah}

\section{Bartramia longicauda (Bechstein, 1812)}

Figure 4A

New records. One male foraging in the flooded grassland of the ESNR $\left(04^{\circ} 56^{\prime} 52^{\prime \prime} \mathrm{N}, 073^{\circ} 57^{\prime} 47^{\prime \prime} \mathrm{W}\right)$ on 15 June 2016. One male foraging around the wetlands of the ESNR $\left(04^{\circ} 56^{\prime} 50^{\prime \prime} \mathrm{N}, 073^{\circ} 57^{\prime} 16^{\prime \prime} \mathrm{W}\right)$ on 14 January 2017. One male foraging on the beach of the wetlands of the ESNR $\left(04^{\circ} 56^{\prime} 55^{\prime \prime} \mathrm{N}, 073^{\circ} 57^{\prime} 22^{\prime \prime} \mathrm{W}\right)$ on 19 January 2018. We recorded this species in the systematic survey on 17 January 2019.

Identification. Individuals brown mottled, with long neck, small head, and large eyes.

Remarks. We did not record any reproductive events. This species is a boreal migratory bird.

Distribution. Canada to Argentina.

\section{Caracara cheriway Jacquin, 1784}

Figure 4B

New records. One adult perching in Pinus sp. in an exotic forest on the border of the ESNR $\left(04^{\circ} 56^{\prime} 43^{\prime \prime} \mathrm{N}, 073^{\circ}\right.$ $57^{\prime} 32^{\prime \prime} \mathrm{W}$ ) on 15 June 2016 . One individual flying and foraging on the grassland of the ESNR $\left(04^{\circ} 56^{\prime} 52^{\prime \prime} \mathrm{N}, 073^{\circ}\right.$ $57^{\prime} 47^{\prime \prime} \mathrm{W}$ ) on 14 January 2017. One individual flying and foraging on the grassland of the ESNR $\left(04^{\circ} 56^{\prime} 48^{\prime \prime} \mathrm{N}\right.$, $073^{\circ} 57^{\prime} 14^{\prime \prime} \mathrm{W}$ ) on 19 June 2018 . We recorded this species in the systematic survey on 17 January and 15 Feb. 2019.

Identification. Similar to a hawk, with sharp beak and claws. The body is black with white and strongly yellow (adult) or grey (young) legs. The dorsal region of the head is black, contrasting with the white neck and the yellow-orange face. Young individuals tend to be brown but darken to black with age.

Remarks. We did not record any reproductive events. Distribution. Florida and southwestern USA, Caribbean, and Central America, south to northern South America.

\section{Charadrius vociferus Linnaeus, 1758}

Figure 4C-E

New records. Five individuals foraging and vocalizing on the open grounds and grasslands of the ESNR $\left(04^{\circ} 56^{\prime} 52^{\prime \prime} \mathrm{N}, 073^{\circ} 57^{\prime} 47^{\prime \prime} \mathrm{W}\right)$ on 15 June 2016 . One individual flying over the grasslands of the ESNR $\left(04^{\circ}\right.$ $\left.56^{\prime} 58^{\prime \prime} \mathrm{N}, 073^{\circ} 57^{\prime} 33^{\prime \prime} \mathrm{W}\right)$ on 17 September 2019. We recorded this species in all the samples of the systematic survey since 19 January 2017.

Identification. Male brown on the back and white ventrally, with two black bands on the chest and black and white spots on the face. Immature individuals with attenuated or incomplete pectoral bands and light red eye rings. Tibial feathers with presence of down. Light brown on edge of wing coverts.

Remarks. We observed three immature individuals, which were flightless and likely less than 30 days. On 21 May, we recorded a nest with two eggs $(35.4 \mathrm{~mm} \times$ $26.1 \mathrm{~mm}$ ) and a chick on 27 May 2018. We also observed three 10-day-old chicks.

Distribution. Canada to northern Colombia, northwestern Venezuela, and western Ecuador and Peru.

\section{Myiodynastes luteiventris P.L. Sclater, 1859 \\ Figure 4F}

New records. One individual perching on a tree crown in the exotic forest of the ESNR (04 $\left.56^{\prime} 52^{\prime \prime} \mathrm{N}, 073^{\circ} 57^{\prime} 47^{\prime \prime} \mathrm{W}\right)$ on 15 June 2016. One individual perching on Smallanthus

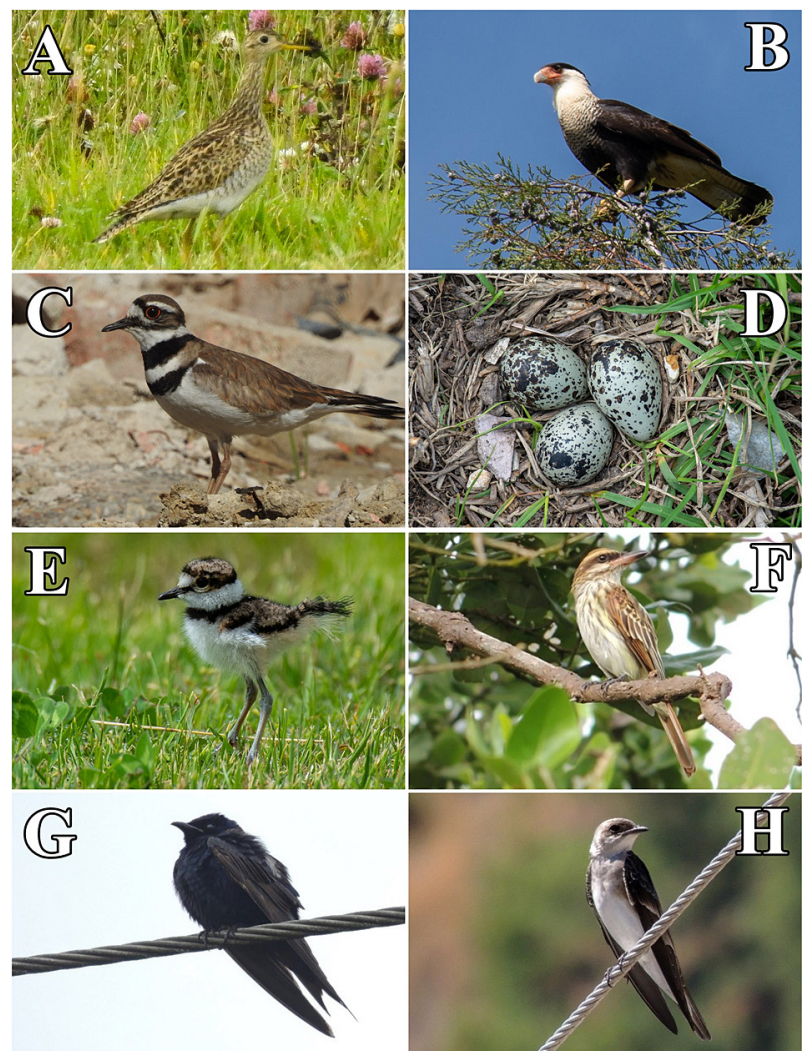

Figure 4. New records from the Bogotá region. A. Bartramia longicauda. B. Caracara cheriway. C. Charadrius vociferus D. Breeding evidence of the migratory bird C. vociferus. E. C. vociferus chick. F. Myiodynastes luteiventris. G. Progne subis male. H. P. tapera. 
pyramidalis (Triana) $\mathrm{H}$. Rob. in the growing native forest of the ESNR $\left(04^{\circ} 56^{\prime} 57^{\prime \prime} \mathrm{N}, 073^{\circ} 57^{\prime} 20^{\prime \prime} \mathrm{W}\right)$ on 14 January 2017. We recorded this species in systematic surveys on 17 January 2018 and on 19 January 2019.

Identification. White eyebrows and whiskers. Glossy ruffle tail and yellowish chest with black lines and blackish whiskers.

Remarks. We did not record any reproductive events. This species has rarely been seen in Colombia and in the Bogotá Savannah. This is a boreal migratory species.

Distribution. Southeastern Arizona (USA), northeastern Mexico, south to Costa Rica and western Amazonia in South America.

\section{Progne subis Linnaeus, 1758}

Figure 4G

New records. Three males flying above the wetlands of the ESNR $\left(04^{\circ} 56^{\prime} 52^{\prime \prime} \mathrm{N}, 073^{\circ} 57^{\prime} 47^{\prime \prime} \mathrm{W}\right)$ on 15 January 2016. Two male flying above the artificial water bodies in the Jaime Duque Park (045 $\left.6^{\prime} 47^{\prime \prime} \mathrm{N}, 073^{\circ} 57^{\prime} 41^{\prime \prime} \mathrm{W}\right)$, 14 January 2017. One male perching on electrical wires around artificial water bodies in the Jaime Duque Park $\left(04^{\circ}\right.$ $56^{\prime} 40^{\prime \prime} \mathrm{N}, 073^{\circ} 57^{\prime} 33^{\prime \prime} \mathrm{W}$ ) on 17 January 2019 . We recorded this species in systematic surveys on 15 October 2018.

Identification. Male with lustrous blue body. Female and immature with greyish front, purple-brown back on head and wings, greyish brown throat and chest, grey sides of neck and nape with darker cheek, and white bottoms, striated with brown.

Remarks. We did not record any reproductive events. This species is a boreal migratory bird.

Distribution. Eastern and central USA to southeastern Brazil.

\section{Progne tapera Linnaeus, 1766}

Figure $4 \mathrm{H}$

New records. Four individuals flying above the wetlands of the ESNR $\left(04^{\circ} 56^{\prime} 52^{\prime \prime} \mathrm{N}, 073^{\circ} 57^{\prime} 47^{\prime \prime} \mathrm{W}\right)$ on 05 January 2018. One individual perching on electrical wires and five individuals flying above the wetlands in the ESNR $\left(04^{\circ} 56^{\prime} 45^{\prime \prime} \mathrm{N}, 073^{\circ} 57^{\prime} 24^{\prime \prime} \mathrm{W}\right)$. We recorded this species systematically for the first time on 17 January 2018, and since then we have observed it between September and December and April and May.

Identification. Large broad-winged swallow; greybrown back with blackish front and helms, white ventral region with grey-brown broadband and a longitudinal line of fusca spots; white and long infracaudal covers contrast with the dark grey tail; immature grayer and without a marked longitudinal line.

Remarks. We did not record any reproductive events. This species is an austral migratory bird.

Distribution. Panama to Argentina.

Setophaga cerulea (Wilson, 1810)

New records. One male perching on Sambucus sp. in the growing native forest of the ESNR $\left(04^{\circ} 56^{\prime} 52^{\prime \prime} \mathrm{N}\right.$, $\left.073^{\circ} 57^{\prime} 47^{\prime \prime} \mathrm{W}\right)$ on 14 January 2016. One male foraging near Eucalyptus sp. in the exotic forest in the ESNR $\left(04^{\circ}\right.$ $56^{\prime} 59^{\prime} \mathrm{N}, 073^{\circ} 57^{\prime} 22^{\prime \prime} \mathrm{W}$ ) on 17 January 2019 . We recorded this species in systematic surveys on 17 January 2017.

Identification. Adult males with cerulean blue dorsal region, blackish wings with two white wing bars; back striated with black; white ventral region with narrow black pectoral collar and blackish striatum on the sides. Female and immature with olive-green back and blue on the crown; narrow superciliary and two white wing bars; opaque white ventral region with dark brown striatum on the lateral sides.

Remarks. We did not record any reproductive events. This species is a boreal migratory bird. It is categorized as Near Threatened (BirdLife International 2019). This species has undergone a population decline of $72 \%$ over the last 44 years in North America (Rosenberg et al. 2016).

Distribution. Eastern USA and Central America, Panama, northern Venezuela, northern and central Colombia, Ecuador, Peru, and northern Bolivia.

\section{Syrigma sibilatrix Temminck, 1824}

New records. Two individuals foraging on the edge of the wetlands in the ESNR $\left(04^{\circ} 56^{\prime} 52^{\prime \prime} \mathrm{N}, 073^{\circ} 57^{\prime} 47^{\prime \prime} \mathrm{W}\right)$ on 15 June 2016. One individual foraging among the aquatic vegetations in the wetlands of the ESNR $\left(04^{\circ} 57^{\prime} 01^{\prime \prime} \mathrm{N}\right.$, $073^{\circ} 57^{\prime} 17^{\prime \prime} \mathrm{W}$ ) on 14 June 2017 . We recorded this species in systematic surveys on 17 January 2017 and on 15 September 2018.

Identification. Black crown, sides of the head, neck, and chest; scapular area suede coloured.

Remarks. We did not record any reproductive events.

Distribution. Llanos grasslands of Venezuela and eastern Colombia; southeastern South America, from the Pantanal of Bolivia south to southeastern Brazil, Uruguay, and northeastern Argentina.

\section{Endangered and Near Threatened species}

\section{Rallus semiplumbeus P.L. Scatler, 1856}

Figure 5A

New records. One individual was heard and seen, hiding among the aquatic vegetations in the wetlands of the ESNR on 2 June 2019. We recorded this species in the systematic surveys on 17 June 2019.

Identification. Males and females have a long, bright red bill and red tarsi, upperparts olive brown, streaked with black or dusky, wings chestnut, sides of the head, neck, and the breast and upper belly are gray, flanks and lower belly are barred black and white.

Remarks. We did not record any reproductive events. The species is very rare in the Bogotá Savannah. It is categorized as Endangered because its range is very small and is contracting owing to widespread habitat loss and degradation (Birdlife International 2016). 
Distribution. This species is reported to eastern Andes of Colombia only.

\section{Gallinago nobilis P.L. Scater, 1856}

Figure 5B, C

New records. Several adults and chicks have been seen flying and foraging in the flooded grasslands of the ESNR. We recorded this species in all samples of the systematic surveys since 13 April 2017.

Identification. Beak long, up to twice the length of the head. Body speckled with black, with darker neck and chest. Central region of the belly white.

Remarks. We saw courtship behavior at the beginning of May and until the end of June 2018. We found three active nests (27 May, 12 June, and 9 July 2018) in flooded grassland, each with two eggs $(47.1 \mathrm{~mm} \times 33.6$ $\mathrm{mm}$ ). This species is mainly threatened by habitat degradation (Birdlife International 2018), and the populations in and around Bogotá have undergone a slight reduction in abundance over the last two decades as a result of a reduction in the availability of shoreline in wetlands due to invasive macrophytes (Stiles et al. 2017). The presence of this species in protected areas in Ecuador (CisnerosHeredia 2006) and Chingaza Natural National Park, Colombia (Linares-Romero et al. 2017), and now in ESNR and PJDNR, where macrophyte invasion is under control, may help increase populations of this bird species.

Distribution. Southwestern Venezuela to southern Ecuador.

\section{Sturnella magna Linnaeus, 1758}

Figure 5D

New records. Several adults and chicks have been heard and seen, hiding in the grasslands of the ESNR. We recorded this species in systematic surveys in all the samples since the beginning of our study.

Identification. Adults with a sturdy body, long pointed beak, long legs, and short tail; head with broad blackish and whitish brown lists; the dorsal region is dark-brown, barred with black; throat, chest, and belly bright yellow with a black V-shaped spot; ventral region dark, mottled brown in young birds.

Remarks. On 10 July 2018, we observed a nest with three chicks devoid of feathers and down and with closed eyes. On 31 October 2018, we recorded a nest with three eggs $(27.74 \mathrm{~mm} \times 20.11 \mathrm{~mm})$. The species is threatened by agricultural activities (use of pesticides and herbicides) and livestock. However, the population trend is currently increasing (Birdlife International 2018). In Bogotá and its surroundings, populations have been undergoing a reduction in their abundance as a result of the loss of grasslands for housing and exotic flower crops (Stiles et al. 2017). As part of the conservation strategy for this species, grassland areas without contaminants where the species can nest can be increased (Jaster et al. 2012).

Distribution. North and Central America and northeastern South America.

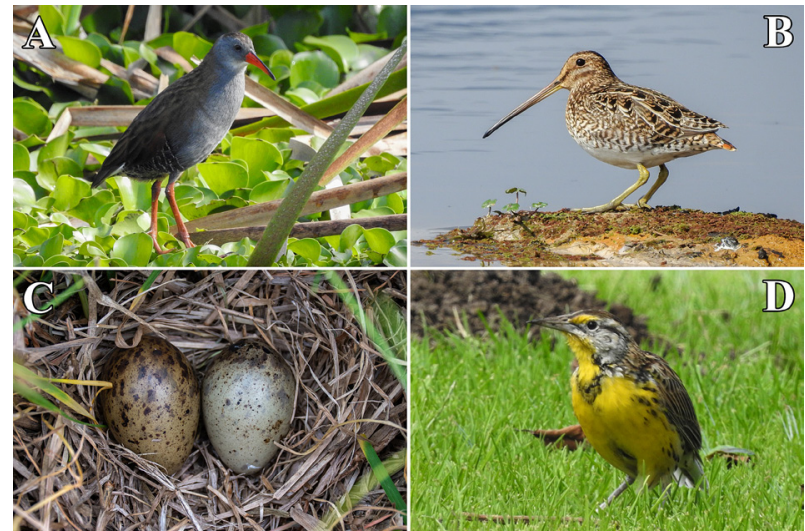

Figure 5. Birds of Bogotá's Savannah. A. Rallus semiplumbeus. B. Gallinago nobilis. C. Breeding evidence of G. nobilis in ESNR. D. Sturnella magna.

\section{Contopus cooperi Nuttall, 1831}

New records. One individual was perching on the treetop of a very conspicuous Eucalyptus sp. We recorded this species in systematic surveys on 4 April 2018.

Identification. Contuopus cooperi is the largest of the pewees, with heavy grayish markings on the sides as if the bird is wearing a waistcoat.

Remarks. We did not record any reproductive events. This species is a boreal migratory bird. It is threatened by logging and pollution from agricultural activities (Birdlife International 2017), as well as loss and alteration of its habitat (Altman 1997). The populations in and around Bogotá have been unchanged over the past 20 years (Stiles et al. 2017).

Distribution. Canada to northern Bolivia and Brazil.

\section{Other noteworthy records}

\section{Anas andium Conover, 1941}

Figure 6A

New records. Three individuals were inside a flock of Spatula discors in the wetlands of the ESNR. We recorded this species in systematic surveys on 19 December 2018 .

Identification. This teal species is similar to the female of Spatula discors (Blue-winged Tail), but darker, with green speculum and orange lower secondary covers.

Remarks. We did not record any reproductive events.

Distribution. Andes of southwestern Venezuela, Colombia, Ecuador, and northern Peru. In Colombia, east of the Andes to Bogotá, between 2,600 and 4,300 m a.s.l.

\section{Oxyura jamaicensis andina Gmelin, 1789 \\ Figure 6B-D}

New records. One male and one female swimming in the wetlands of the ESNR on 21 June 2017. We recorded this species in all systematic surveys since 16 July 2017. Identification. Small and chubby duck with an upward turned tail. Male has black head and neck with a varied amount of white on the cheek. Bill light blue. Female blackish. 
Remarks. We recorded three reproductive events. Th first event was on 25 May 2018, with five chicks; the second event was on 6 June 2018, with four chicks; and the last event occurred on 20 September 2018, when we found one nest with four eggs $(68.3 \mathrm{~mm} \times 48.1 \mathrm{~mm})$.

Distribution. North America, Mexico, and Colombia. In South America, northern Andean mountains to southern Argentina and Chile. In Colombia at elevations between 2,500 and 4,000 $\mathrm{m}$ a.s.l.

\section{Spatula discors Linnaeus, 1766}

Figure 6E-H

New records. One female was walking on flooded grasslands of the ESNR on 24 June 2016. We recorded this species in all systematic surveys since 17 July 2016.

Identification. Males with grey head and white crescent on the face and flanks. The covering feathers make blue patches. Females with mottled brown head and neck, but without half-moon.

Remarks. We recorded a female and eight chicks swimming in a wetland in the ESNR on 30 July 2016. Between 21 April and 12 June 2018, we recorded 11 active nests with an average of 11 eggs $(45.5 \mathrm{~mm} \times 33 \mathrm{~mm})$, of which $83 \%$ of chicks hatched, a high value compared to $35 \%$ reported by Glover (1956). A boreal migratory species.

Distribution. North America through Central America to northern South America, Uruguay, Argentina, and Chile.

\section{Lesbia nuna Lesson, 1832}

Figure 7A

New records. One male and one female sucking flower nectar of D. mutisii and Streptosolen jamesonii (Benth.) Miers in the native forest in ESNR on 20 October 2018. We recorded this species in systematic surveys on 16 December 2018 and on 15 April 2019.

Identification. Male bright green male ventrally; tail length approximately twice the length of the body; black helms and their distal half bright green. Females with bright green spots on white ventral region and shorter tail than in males.

Remarks. We did not record any reproductive events. The species was rare in our study.

Distribution. Colombia, Ecuador, and Peru.

\section{Buteo platypterus Vieillot, 1823}

Figure 7B, C

New records. About 1,500 individuals were recorded in a boreal migration flying above the ESNR and PJDNR on 7 April 2018. The flock included Buteo platypterus and B. swainsoni Bonaparte, 1838. We recorded this species in systematic surveys in November of each year.

Identification. Adult and immature individuals show differences in plumage. Adults have dark brown on the dorsum and barred chest. Immatures are similar on dorsum, but the chest is whitish with brown stripes.

Remarks. We did not record any reproductive events. This species is a boreal migratory bird.

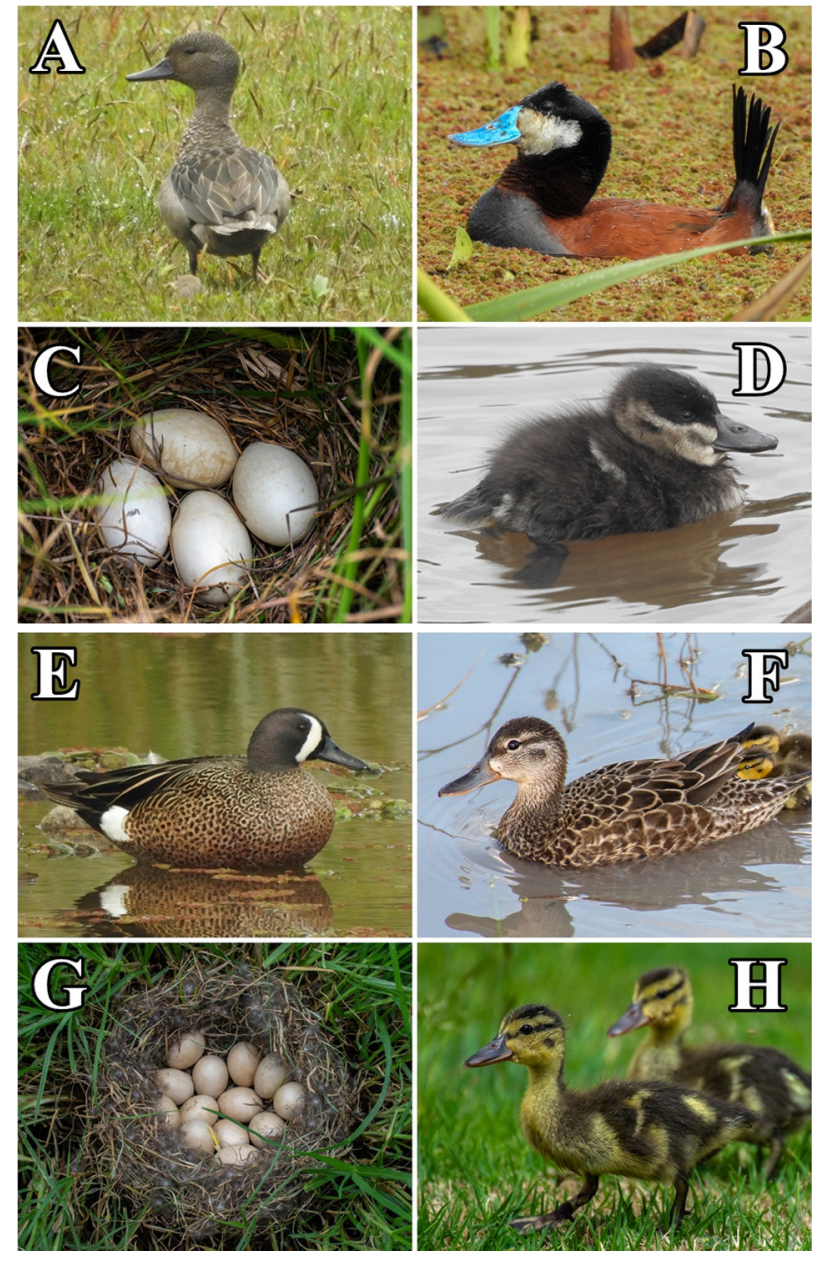

Figure 6. Birds of Bogotá's Savannah. A.Anas andium. B. Oxyura jamaicensis andina male. C. Breeding evidence of $O$. jamaicensis andina in ESNR, nest and eggs. D. O. jamaicensis andina chick. E. Spatula discors male. F. S. discors female. G. Breeding evidence of migratory bird S. discors in ESNR, nest and eggs, H. S. discors chicks.

Distribution. USA, Canada, Mexico, Central America, northwestern South America to western Ecuador, Bolivia, and northwestern Brazil.

\section{Elanoides forficatus Linnaeus, 1758}

New records. Three individuals flying over PJDNR on 12 June 2016.

Identification. The body is a contrast between deep black and white. The flight feathers, tail feet and bill are black. The tail is elongated and forked.

Remarks. We did not record any reproductive events. This species is a boreal migratory bird. We consider this species as an isolated record due to its unique record of three individuals. They could be wandering individuals of the subspecies Elanoides forficatus yetapa (Vieillot, 1818) resident in Colombia (Ayerbe-Quiñones 2018), being recorded at a time when the migration of birds from the north of the continent was not usual.

Distribution. Southeastern USA south through Central America and southern Brazil.

Falco peregrinus Tunstall, 1771

Figure 7D 


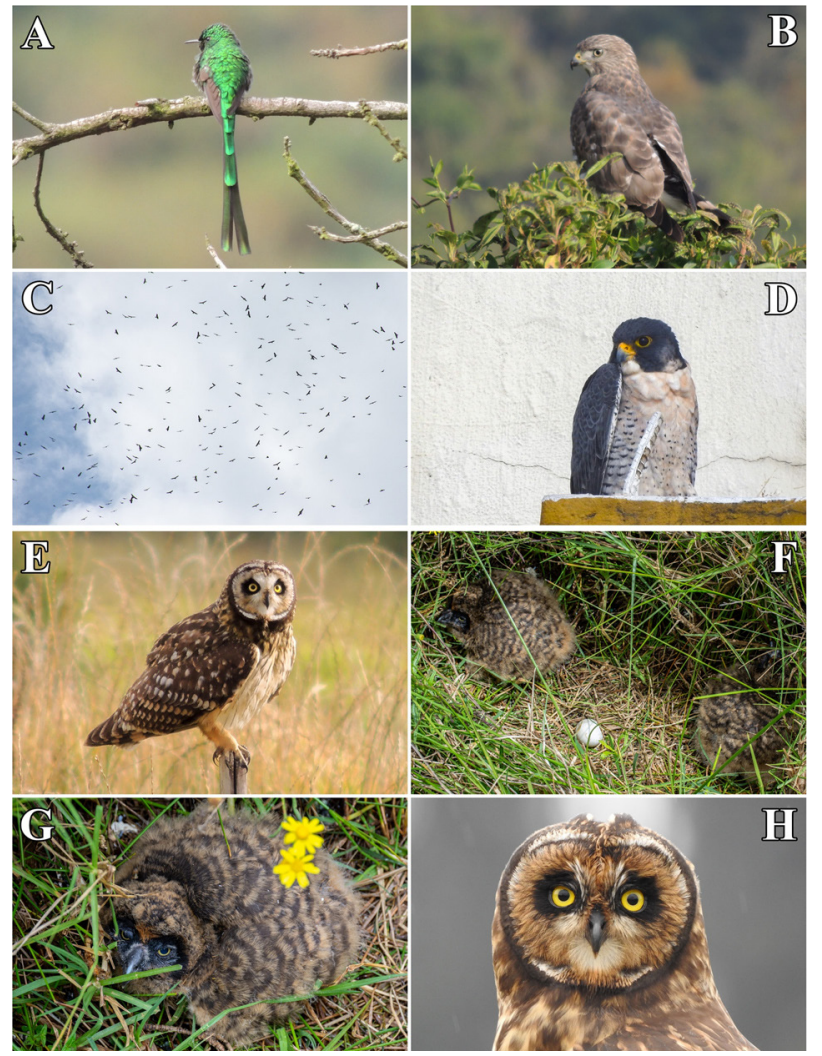

Figure 7. Birds of Bogotá's Savannah. A. Lesbia nuna male, B. Buteo platypterus. C. Migratory flock of B. platypterus and B. swainsoni. D. Falco peregrinus. E-H. Asio flammeus bogotensis. E. Adult. F. Chicks and egg. G. Chick. H. Immature.

New records. Lonely individuals and couples have been seen perching and feeding on doves and pigeons in a replica building of the Taj Mahal. We recorded this species systematically between October 2018 and April 2019.

Identification. Dorsal region of the body is dark bluegrey, with the crown, nape and broad legs under the eye black. Atrial region, lateral areas and lower parts of the neck are white. Chest and belly in brown are barred, with brown tailed tail, grey blue and greyish white. Head sides, including black ears.

Remarks. We did not record any reproductive events. This species is a boreal migratory bird. It is a winter resident scarce in the Bogotá Savannah (ABO 2000).

Geographic distribution. Western North America, Central and South America.

\section{Asio flammeus bogotensis Chapman, 1915}

Figure 7E-H

New records. One individual perching on an artificial hanger of $2 \mathrm{~m}$ in the ESNR on 12 October 2017.We recorded this species systematically on 17 October 2017, 15 November 2017, 14 June 2018, 15 September 2018, 15 January 2019, 17 April 2019.

Identification. Owl with short ears, feathered legs and dark facial disc until black around the eyes. Whiskers, nostrils and brows are blackish dorsally. Tawny lists, beak, and black claws. During flight, it shows a black patch on the wrists and above it an extensive light coloured patch at the base of the front.

Remarks. On 16 July 2018. we recorded a nest with two chicks of $300 \mathrm{~g}$ and an egg with embryonic death (40.1 $\mathrm{mm} \times 33.9 \mathrm{~mm}$ ). The species was very common in open areas of the Bogotá Savannah, currently very rare (ABO 2000; Stiles et al. 2017).

Distribution. Colombia. Cosmopolitan distribution.

\section{Fulica americana Gmelin, 1789}

Figure 8A-D

New records. One individual swimming, four months after the increase of the open water habitat in the wetlands of the ESNR on 2 June 2016. We recorded this species systematically in all samples since 17 June 2016.

Identification. Short and thick beak (turns yellow during reproduction) with an incomplete black ring towards the end. Small brown shield, grey body with black head and neck. Lateral regions and white infra-feather feathers.

Remarks. We recorded three nests on 6 October 2018, 1 Feb. 2019, and 11 Mar. 2019. In the nests, we found four eggs $(51.8 \mathrm{~mm} \times 35.1 \mathrm{~mm})$. The building material of the floating nests includes aquatic vegetation such as Bidens laevis (L.) Britton, Stern \& Poggenb., 1888 (Larger Bur-Marigold), and Schoenoplecptus californicus (Mey.) Soják, 1972 (California Bulrush).

Distribution. North and Central America, Caribbean, and in northern South America.

\section{Gallinula galeata Lichtenstein, 1818}

Figure 8E-H

New records. Two individuals foraging in the aquatic vegetation, four months after the increase of the open water habitat in the wetlands of the ESNR on 17 June 2016. We recorded this species systematically in all samples since then.

Identification. Black chicks with frontal shield and light red beak, and light grey-brown young individuals in the ventral region with grey on the head and neck; body with a lateral white stripe; charcoal grey adults, with side stripe and white tail feathers, red forehead shield and red beak with yellow tip.

Remarks. We saw a nest hidden in the vegetations on $11 \mathrm{Mar}$. 2019. It had five eggs $(44.5 \mathrm{~mm} \times 31.7 \mathrm{~mm})$. The building material of the floating nest includes $B$. laevis and Ludwigia peploides (Kunth) P. H. Raven, 1963 (Floating Primrose-Willow), and Cenchrus clandestinus (Hochst. ex Chiov.) Morrone.

Distribution. Eastern and southern Canada to northern Argentina.

\section{Porphyriops melanops bogotensis (Vieillot, 1819) Figure 9A-D}

New records. Two individuals hiding in the aquatic vegetation of the wetland in ESNR on 07 December 2016. We recorded this species systematically in all samples since 14 December 2016. 


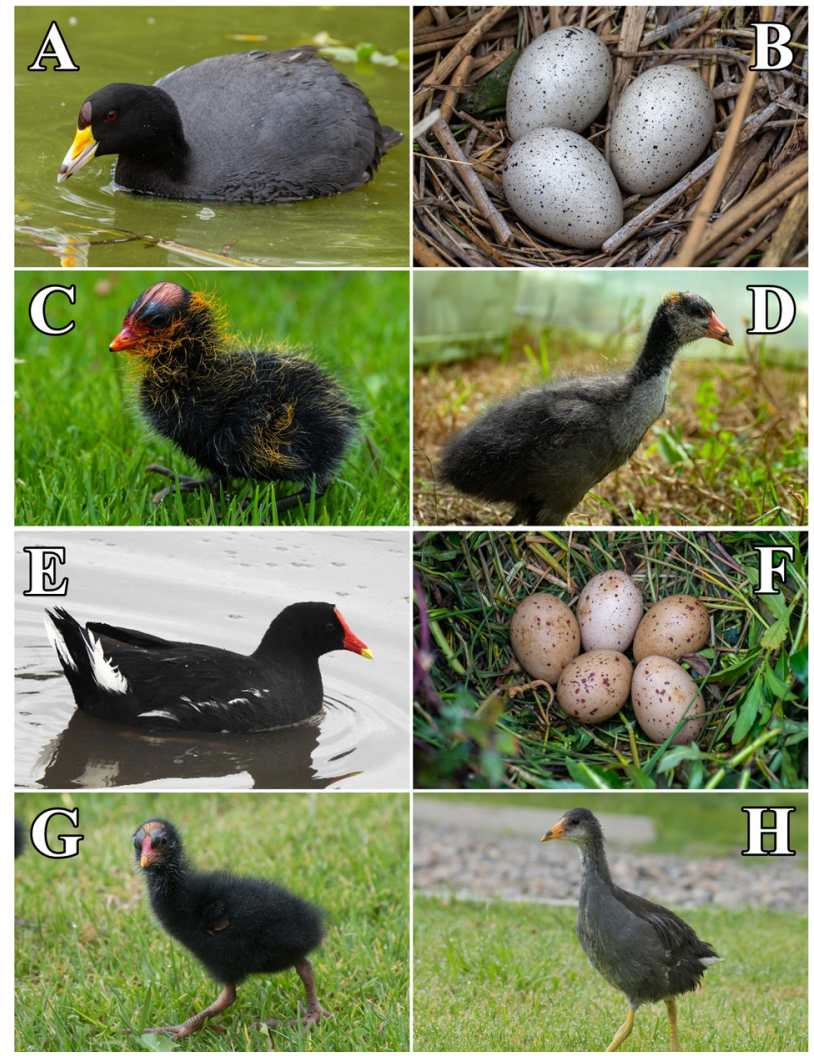

Figure 8. Birds of Bogotá's Savannah. A-D. Fulica americana. A. Adult. B. Breeding evidence of eggs in ESNR. C. Chick. D. Immature. E-H. Gallinula galeata. E. Adult. F. Breeding evidence of eggs in ESNR. G. Chick. H. Immature.

Identification. Black chick with red beak; young brown body, darker towards the back, throat and chest. Adults with light green beak with blackish crown; head, back and supracaudal coverts of olive-brown colour; dark grey neck and chest; dark brown and white mottled flanks; abdomen and infracaudal coverts of white colour.

Remarks. During the study time we recorded 17 active nests with three to six eggs $(40.8 \mathrm{~mm} \times 28.8 \mathrm{~mm})$. The nests were near to the water's edge, hidden in the vegetations or floating in the water. Nest building material includes aquatic vegetation such as $B$. laevis, S. californicus, and Ciperus papirus Linnaeus, 1753 (Papyrus Sedge). Several individuals have been seeing diving and swimming as an escape strategy. This species has been locally categorized as endangered danger (MADS 2017). In Bogotá and its surrounding, populations are threatened by urbanization and loss of habitat (Stiles et al. 2017). Conservation strategies for this species require alternatives such as those followed in the reserves under study, including the construction of artificial wetlands, where some populations appear to survive on the banks of the Bogotá River (Stiles et al. 2017).

Distribution. Center Colombia, northeastern and southwestern Brazil, southern Bolivia, northern Paraguay, eastern Argentina and Chile.

\section{Actitis macularius Linnaeus, 1766}

Figure 9E

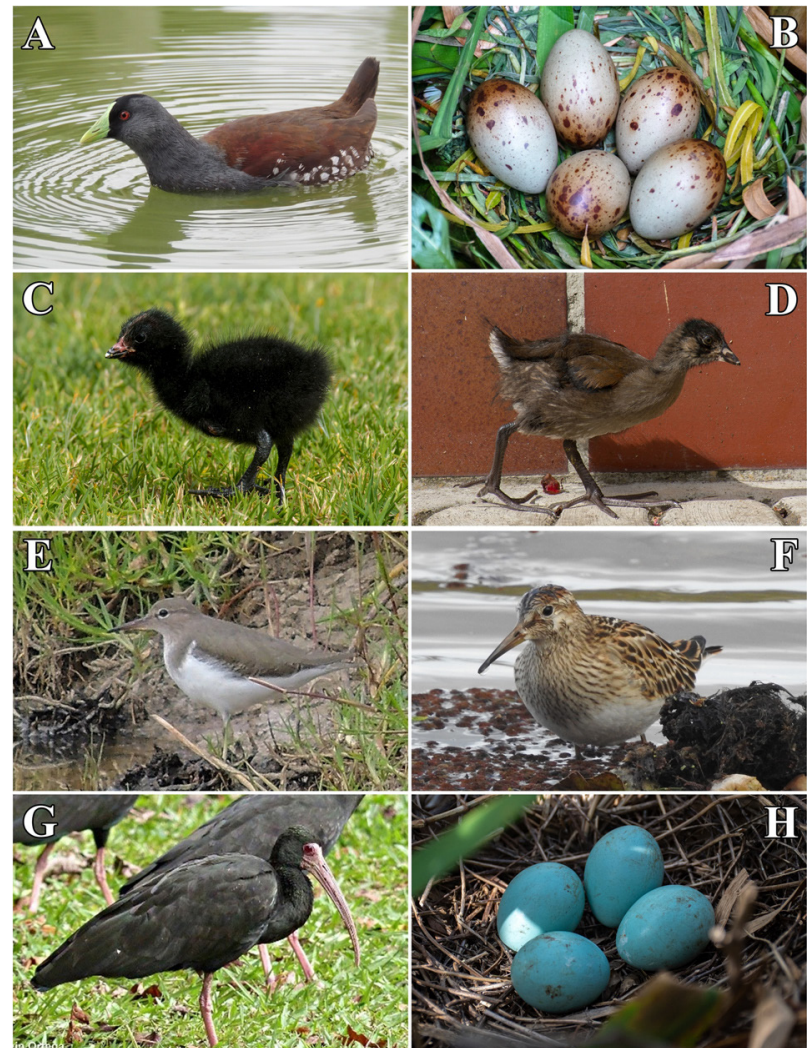

Figure 9. Birds of Bogotá's Savannah. A-D. Porphyriops melanops bogotensis. A. Adult. B. Breeding evidence of eggs in ESNR. C. Chick. D. Immature. E. Actitis macularius. F. Calidris melanotos. G. Phimosus infuscatus. H. Breeding evidence of $P$. infuscatus eggs in ESNR.

New records. Individuals with breeding or reproductive plumage, as well as individuals with winter plumage, have been seen. We recorded this species systematically in all samples during most of the year from August to May.

Identification. It is the smallest species of sandpipers in the Bogotá Savannah. Dorsal region of brown colour, with the coverts barred. Eye list and chest sides are brown, whitish eyebrows. White ventral region (non-reproductive) and mottled (reproductive). Legs are yellow.

Remarks. We did not record any reproductive events. This species is a boreal migratory bird.

Distribution. From Canada to Chile and northern Argentina.

\section{Calidris melanotos (Vieillot, 1819)}

Figure 9F

New records. An individual foraging at the edge of the wetland in the ESNR on 10 Feb. 2019. It remained in the area for at least a week. We recorded this species systematically on 17 Feb. 2019.

Identification. Dark breast streaking which stops at a bold clean break on the breast, or pectoral area.

Remarks. We did not record any reproductive events. This species is a boreal migratory bird scarce in the Bogotá Savannah (ABO 2000).

Distribution. Alaska to southern South America. 
Phimosus infuscatus Lichtenstein, 1823

Figure 9G, H

New records. One individual flying over a patch of aquatic vegetation composed mainly of $T$. latifolia in the ESNR on 06 June 2018. In its beak the bird carried sticks as a possible nest building material. We recorded this species systematically on 17 Feb. 2018, 14 Aug. 2018, 15 January 2019, 15 Feb. 2019, 15 Mar. 2019 and 17 April 2019.

Identification. Red face with pink-coloured beak with bare facial skin, reddish legs and blackish greenish plumage.

Remarks. The species is abundant in other wetlands in the region, but in our study area only between one to three individuals were recorded.

Distribution. Northern Colombia east through the Venezuelan llanos, eastern Brazil west to Bolivia and south to central Argentina.

\section{Sicalis luteola bogotensis Sparrman, 1789}

Figure 10A

New records. Several individuals foraging in the grasslands of the ESNR on $14 \mathrm{Feb}$. 2016. We recorded this species systematically in all samples since then.

Identification. Light olive dorsal region with striated brown; fluted brown crown; tailbone and sides of the head yellowish olive; wings and tail dark brown, lighter towards the edge; flanges and bright yellow eye area; female similar to the male but clearer.

Remarks. Between February and May, males are recorded flying energetically over the grasslands while vocalizing, a behavior associated with courtship.

Distribution. Southern Mexico to Argentina and Chile.

\section{Synallaxis subpudica P.L. Sclater, 1874}

Figure 10B

New records. One individual hiding on the shrubby vegetation of the wetland in the ESNR on 15 December 2018. We recorded this species systematically on the same date and it is our unique record in the ESNR.

Identification. Adults with long spine; brown back; crown and part of the wings of rufous colour, greyish brown tail, belly and throat whitish brown; speckled throat with black, young ones are similar to adults, but without crown and wings with little ruff.

Remarks. This species is very common in the PJDNR. It is endemic from the eastern cordillera. We recorded a nest in PJDNR on April 27, 2019. It was built with prickly sticks shaped like a ball, inside a white egg.

Distribution. From the north area of Boyacá to Bogotá.

Chrysomus icterocephalus bogotensis Linnaeus, 1766 Figure 10C, D

New records. Several individuals flying around the wetland vocalizing around the wetlands of the ESNR on 27 June 2016. Since then, we have recorded this species

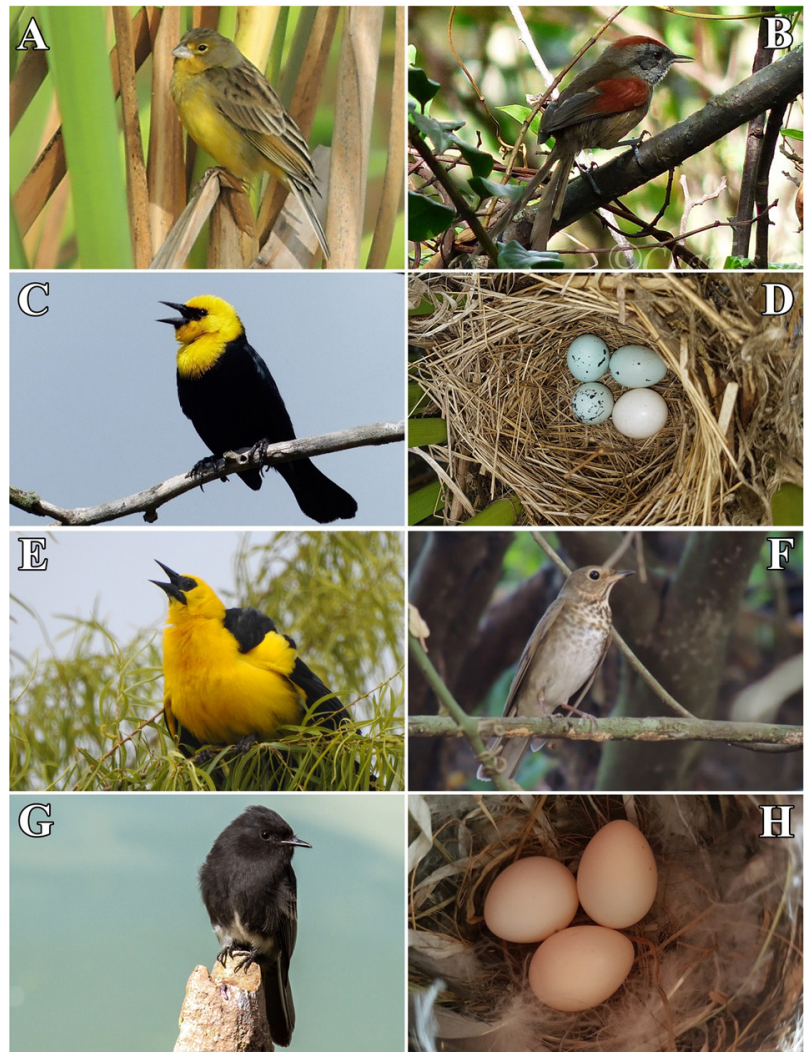

Figure 10. Birds of Bogotá's Savannah. A. Sicalis luteola bogotensis male. B. Synallaxis subpudica. C. Chrysomus icterocephalus bogotensis male. D. Breeding evidence of $C$. icterocephalus bogotensis parasitized by Molothrus bonariensis in ESNR. nest and eggs. E. Gymnomystax mexicanus. F. Catharus ustulatus. G. Sayornis nigricans. H. Breeding evidence of S. nigricans eggs in ESNR.

systematically in over $80 \%$ of the samples.

Identification. Adult males with black body and yellow hood with black flanges; conical peak; female with brown olive dorsal region with diffuse brown striatum; superciliary and yellow throat, with facial area and opaque yellow crown, rest of the olive yellow body; young ones similar to females but paler tending to whitish.

Remarks. On 16 June 2018, we observed a couple feeding a young individual of Molothrus bonariensis Gmelin, 1789. On 08 April 2019. we recorded two cup-shaped nests with light blue eggs $(22.8 \mathrm{~mm} \times 16.9 \mathrm{~mm})$ with some dark spots in its widest part. The population has been increasing since 2017 after the restoration actions carried out in the wetlands.

Distribution. Colombia to northern Brazil and northeastern Peru.

\section{Gymnomystax mexicanus Linnaeus, 1766} Figure 10E

New records. An adult being attacked by individuals of Chrysomus icterocephalus. bogotensis during the latter's breeding season on 15 May 2018. The species was recorded systematically on 17 May 2018.

Identification. Adults with robust beaks; golden yellow body with back, wings, tail, black flanges and eye area; yellow triangular spot in the basal region of the wings. 
Remarks. We did not record any reproductive events. This species is a visitor that came from warmer lowlands (Stiles et al. 2017).

Distribution. Colombia, Venezuela, northern French Guiana, Brazil and Peru.

\section{Catharus ustulatus Nuttall, 1840}

\section{Figure 10F}

New records. One individual flying around the native plantations in the ESNR on 14 April 2018. Since then, it has been seen between October and May with greater abundance (up to 20 individuals) in mid-October. We recorded this species systematically on 17 October 2018. and 14 Feb. 2019.

Identification. Black-spotted chest with large eyes, long thin legs, olive brown back and mottled white chest with brown, light brown eye ring, brown head sides with lighter anterior area, black peak with pink basal region, pink legs; immatures similar to adults but with dark brown terminal spots.

Remarks. We did not record any reproductive events. This species is a boreal migratory bird.

Distribution. From Alaska and northern Canada to northern Argentina.

\section{Sayornis nigricans Swainson, 1827}

Figure 10G, H

New record. One individual flying and foraging about the wetlands in the ESNR on 15 September 2016.

Identification. Body small with a large head and a medium-long squared tail, dark sooty gray overall with a white belly.

Remarks. On Aug. 30, 2019. We recorded a cup-shaped nest built with mud, feathers and hair under a concrete bridge. We found three eggs $(20.20 \mathrm{~mm} \times 15.41 \mathrm{~mm})$. In ESNR the records of this species are unusual, while for PJDNR it is recorded constantly.

Distribution. Southwestern United States to Central and South America.

\section{Discussion}

We found out that our study reported species not recorded in other localities of the area, with 32\% (41 spp.) not reported for Chingaza PNN, 72\% (93 spp.) not reported for BOBPFR, 71\% (92 spp.) not reported at the wetlands of Bogotá D.C., and 23\% (30 spp.) not reported at any other localities in the area. At the same time, richness in the ESNR changed over the years: in 2016 we recorded 42 species; in 2017, 71 species; in 2018, 92 species, and in 2019, 86 species. The new records in the localities studied and the variation in richness in the ESNR can be related to the diversity of habitats in the reserve as a result of the restoration process. These habitats are the growing native forests, scrubs and wetlands, places where most of the bird species are found in the Bogotá region (Stiles et al. 2017). Other habitats in the study area where we reported several species are the eucalyptus patches and grasslands. All these mosaics of habitats found in the ESNR are located in strategic spots between the basin of the Bogotá river and the Tibito hill.

Another action of restoration was to reduce populations of the invasive macrophyte species (e.g., Eichornia crassipes) that had eutrophicated the two wetlands. Likewise, we reintroduced species of aquatic vegetation as Juncus effusus Linnaeus, 1753 (Lamp Rush), Schoenoplecptus californicus, Persicaria punctata (Elliott) Small, 1903 (Dotted Smartweed), and Bidens laevis, because these vegetations are crucial to the reproduction of some bird species. It is known that the extension of open water habitat and the enrichment of aquatic flora, benefit some species of water birds (Hinojosa-Huerta et al. 2008; Stiles et al. 2017).

For ESNR, threatened subspecies such as Porphyriops melanops bogotensis and Oxyura jamaicensis andina (Renjifo et al. 2016; MADS 2017) and other species, such as Actitis macularius, Gallinula galeata, Fulica americana, Porzana carolina (Linnaeus, 1758), Spatula discors, and Tringa solitaria Wilson, 1813 became more common. Actitis macularius and T. solitaria are associated with beaches and shallow waters with aquatic vegetation (ABO 2000). These requirements began to be fulfilled in 2016 due to the reduction of wetland slope, carried out in places where before there were no beaches.

Stiles et al. (2017) reported that the increase in shrubby vegetations around some wetlands in Bogotá has led to the increment in S. subpudica and T. aedon populations, however, these trends are not clear in the ESNR since $S$. subpudica has been reported just once and $T$. aedon had an occurrence frequency of $81 \%$ in 2016 which increased to $100 \%$ in the posterior years. Synallaxis subpudica inhabits dense shrubs like the ones found in the PJDNR; these shrubs are not totally established in the ESNR, but it can be expected that as restoration efforts progress, the occurrence of this species will increase.

Tyrannidae increased their richness from two spp. (2016) to nine or 10 spp. (2018-2019), with a historical richness of 14 species. The New World warblers (family Parulidae) raise the number of species from four spp. (2017) to eight spp. (2019), with a historical richness of 10 species. The increased richness of these families could be attributed to increases in the diversity of insects (e.g., dipterans and lepidopterans), recorded in this same period of time in ESNR (unpub. data). Individuals of Tyrannidae and Parulidae families have been widely recognized as predators of insects (Hilty and Brown 2001).

Distribution range of $S$. sibilatrix increased $170 \mathrm{~km}$ to the north of the country. It was recorded in Yopal (Casanare department). Meanwhile, other new records from species such as B. longicauda, C. cheriway, C. vociferus, M. luteiventris, P. subis, P. tapera and S. cerulea were expanded to the western of the country, because these species had been recorded in closer localities inside of the Bogotá Savannah. However, these records are 
expected since our study area is in the route of migration of those species.

In the local context of our study, the Tocancipa and Sesquile development and management plan contemplates the execution of productive agricultural, livestock, mining, industrial and urbanization activities that eliminate and displace some native ecosystems, replacing others with Pinus sp. and Eucalyptus sp. plantations. Although the ordinance plan is emphatic in protecting priority locations in the provision of ecosystem services and scenic places, legally the only existing nature reserves in the municipality are the ESNR and PJDNR. The restoration process in these reserves began in 2016 and has included the plantation of at least 50,000 native plants to replace crops of Solanum tuberosum Linnaeus, 1753 (potato), Zea mays Linnaeus, 1753 (corn), C. cladestinus, and the raising of cattle. In addition, the restoration has allowed adapting artificial water bodies and restoring wetlands. The monitoring of this process has mainly focused on avifauna, which increased from 42 species in 2016 when the process began to the current record of 105 species, with 42 resident species.

Conclusion. The richness of bird species of our study area and the number of species not recorded in similar localities could be related to the diversity of habitats evaluated and the restoration actions implemented. Although the restoration objective of our project is to form a native forest, our actions are in the initial stages. Consequently, grasslands and forest in different successional status are common; these aspects could be reflected in the species we recorded. The monitoring of avifauna in changing ecosystems can increase the historical richness and is an elementary tool to know if the restoration actions have good impacts on the ecosystem.

\section{Acknowledgements}

We thank Jaime Duque Park, Ecoparque Sabana Nature Reserve, Grupo de Investigación en Odonatos y otros artrópodos de Colombia (GINOCO), Grupo de Investigación en Biología (GRIB) of the Biology Department at Universidad El Bosque, and the Centro de Investigación en Acarología. This research received grant from the Foundation Jaime Duque Park through its project "Participative Restoration of the Ecoparque Sabana Nature Reserve".

\section{Authors' Contributions}

FC-V, YC-M, DO collected data on the field and identified the specimens. FC-V, YC-M, FP-R contributed to the design of the study and critical revision, adding intellectual content.

\section{References}

Altman B (1997) Olive-sided flycatcher in western North America: status review. US Fish and Wildlife Service, Portland, $81 \mathrm{pp}$.
ABO (Asociación Bogotana de Ornitología) (2000) Aves de la Sabana de Bogotá. Guía de campo. Quebecor Impreandes, Bogotá, 276 pp.

Ayerbe-Quiñones F (2018) Guía ilustrada de la avifauna colombiana. Wildlife Conservation Society, Bogotá, 212 pp.

BirdLife International (2016) Rallus semiplumbeus. The IUCN Red List of threatened species 2016: e.T22692482A93355621. https:// doi.org/10.2305/iucn.uk.2016-3.rlts.t22692482a93355621.en. Accessed on: 2019-11-28.

BirdLife International (2017) Contopus cooperi (amended version of 2016 assessment). The IUCN Red List of threatened species 2017: e.T22699787A110734937. https://doi.org/10.2305/ IUCN.UK.2017-1.RLTS.T22699787A110734937.en. Accessed on: 2019-6-3.

BirdLife International (2018) Sturnella magna. The IUCN Red List of threatened species 2018: e.T22735434A131853051. https://doi. org/10.2305/IUCN.UK.2018-2.RLTS.T22735434A155622113. en. Accessed on: 2019-6-3.

BirdLife International (2019) Setophaga cerulea. The IUCN Red List of threatened species 2019: e.T22721740A153691320. https://doi. org/10.2305/IUCN.UK.2019-3.RLTS.T22721740A153691320.en. Accessed on: 2020-8-27.

Calvachi B (2003) La fauna de los humedales de la majestuosidad de los mastodontes hacia el oportunismo de las ratas. EAAB y Conservación Internacional. In: Güarnizo A, Calvachi B (Eds) Los humedales de Bogotá y la Sabana. Acueducto de Bogotá y Conservación Internacional, Bogotá, 109-138.

Calvachi B (2012) Los ecosistemas semisecos del altiplano cundiboyacense, bioma azonal singular de Colombia, en gran riesgo de desaparición. Mutis. Revista Universidad Jorge Tadeo Lozano 2 (2): 26-59.

Climate-data.org (2018) Clima Tocancipá. https://es.climate-data.org/ america-del-sur/colombia/cundinamarca/tocancipa-45570/. Accessed on: 2019-6-1.

Cisneros-Heredia DF (2006) A preliminary approach to the Snipes (Gallinago) of Ecuador, with remarks on their distribution in Ecuadorian IBAs and its conservation status. WI-WSSG Newsletter 32: 4-11.

Cortés S, Van der Hammen T, Rangel O (1999) Comunidades vegetales y patrones de degradación y sucesión en la vegetación de los cerros occidentales de Chía Cundinamarca-Colombia. Revista de la Academia Colombiana de Ciencias Exactas 23: 529-554.

D'Angelo Neto N, Venturin N, Oliveira-Filho AT, Costa FAF (1998) Avifauna de quatro fisionomias florestais de pequeno tamanho (5-8 ha) no campus da UFLA. Revista Brasileira de Biologia 58 (3): 463-472. https://doi.org/10.1590/S0034-71081998000300011

Echeverry-Galvis A (2015) Censos nacionales de aves acuáticas, herramienta de monitoreo para los humedales de la Sabana de Bogotá. In: Chaparro-Herrera S, Ochoa D (Eds) Aves de los Humedales de Bogotá, aportes para su conservación. Asociación Bogotana de Ornitología, Bogotá, 1-92.

Etter A, Van Wyngaarden W (2000) Patterns of landscape transformation in Colombia, with emphasis in the Andean Region. Ambio 29: 432-439.

Etter A, McAlpine C, Possingham H (2008) Historical patterns and drivers of landscape change in Colombia since 1500: a regionalized spatial approach. Annals of the Association of American Geographers 98: 2-23.

Glover FA (1956) Nesting production of the blue-winged teal (Anas discolor Linnaeus) in Northwest Iowa. Wildlife Management 20: 28-46.

Hilty SL, Brown WL (2001) Guía de las aves de Colombia. Princeton University Press/ American Bird Conservancy-Universidad del Valle-Sociedad Antioqueña de Ornitología, Cali, 1040 pp.

Hinojosa-Huerta O, Iturribarría-Rojas $\mathrm{H}$, Zamora-Hernández E, Calvo-Fonseca A (2008) Densities, species richness and habitat relationships of the avian community in the Colorado River, Mexico. Studies in Avian Biology 37: 74-82. 
Holdridge LR (1967) Life zone ecology. Tropical Science Center. San José, 149 pp.

IDEAM (Instituto de Hidrología, Meteorología y Estudios Ambientales) (2017) Variabilidad diaria de temperatura. http:// www.ideam.gov.co/web/tiempo-y-clima/variabilidad-diariatemperatura. Accessed on: 2019-6-1.

IGAC (Instituto Geográfico Agustín Codazzi) (1996) Diccionario geográfico de Colombia. Tomo 3. Instituto Geográfico Agustín Codazzi, Bogotá, 1865 pp.

Jaster LA, Jensen WE, Lanyon WE (2012) Eastern Meadowlark (Sturnella magna), version 2.0. In: Rodewald PG (Ed.) The birds of North America. Cornell Lab of Ornithology, New York. https:// doi.org/10.2173/bna.160. Accessed on: 2019-6-1.

Linares-Romero LG, Stiles FG, Roselli L, Camargo P, Candil J, Galindo R,

Avellaneda FE, Pulido AR (2017) Guía de aves del Parque Nacional Natural Chingaza. La imprenta editores S.A. Bogotá, 246 pp.

MADS (Ministerio de Ambiente y Desarrollo Sostenible) (2017) Resolución 1912. República de Colombia, Bogotá, 38 pp.

Mora KG (2015) Los agricultores y ganaderos de la Sabana de Bogotá frente a las fluctuaciones climáticas del siglo XVIII. Fronteras de la Historia 20: 15-42.

Neotropical Birds Online (2019) Cornell Lab of Ornithology, Ithaca, NY, USA. https://neotropical.birds.cornell.edu/Species. Accessed on: 2019-11-1.

Olivares A (1969) Aves de Cundinamarca. Universidad Nacional de Colombia, Bogotá, 425 pp.

Palacios M, Safford F (2002) Colombia: país fragmentado, sociedad dividida, su historia. Editorial Universidad de los Andes, Bogotá, 595 pp.

Peraza CA (2011) Aves, Bosque Oriental de Bogotá Protective Forest Reserve, Bogotá, DC, Colombia. Check List 7: 57-63. https://doi. org $/ 10.15560 / 7.1 .57$

Pérez A (2000) Estructura ecológica principal de la Sabana de Bogotá. Sociedad geográfica de Colombia, Bogotá, 37 pp. https://www. sogeocol.edu.co/documentos/est_eco.pdf. Accessed on: 2019-3-1.

Rangel JO (2006) La biodiversidad de Colombia. Palimpsestos 5: 292304.

Rangel JO (2015) La biodiversidad de Colombia: significado y distribución regional. Revista de la Academia Colombiana de Ciencias Exactas, Físicas y Naturales 39 (151): 176-200.

Renjifo LM, Amaya-Villarreal AM, Burbano-Girón J, Velásquez-Tibatá J (2016) Libro rojo de aves de Colombia, Vol. II: Ecosistemas abiertos, secos, insulares, acuáticos continentales, marinos, tierras altas del Darién y Sierra Nevada de Santa Marta y bosques húmedos del centro, norte y oriente del país. Pontificia Universidad Javeriana e Instituto Alexander von Humboldt, Bogotá, 563 pp.

Rodríguez N, Armenteras D, Morales M, Romero M (2006) Ecosistemas de los Andes Colombianos, Bogotá, Colombia. Instituto de Investigación de Recursos Biológicos Alexander von Humboldt, Bogotá, 154 pp.

Rosenberg KV, Kennedy JA, Dettmers R, Ford RP, Reynolds D, Alexander JD, Beardmore CJ, Blancher PJ, Bogart RE, Butcher GS, Camfield AF, Couturier A, Demarest DW, Easton WE, Giocomo JJ, Keller RH, Mini AE, Panjabi AO, Pashley DN, Rich TD, Ruth JM, Stabins H, Stanton J, Will T (2016) Partners in flight landbird conservation plan: 2016 Revision for Canada and Continental United States. Partners in Flight Science Committee. United States Fish and Wildlife Service, Falls Church, 119 pp.

Stiles FG, Rosselli L, De la Zerda S (2017) Changes over 26 years in the avifauna of the Bogotá Region, Colombia: Has climate change become important? Frontiers in Ecology and Evolution 5: 58. https://doi.org/10.3389/fevo.2017.00058. 